\title{
1 Elasticity of dense actin networks produces nanonewton protrusive forces
}

2

3 Marion Jasnin ${ }^{1, *}$, Jordan Hervy², Stéphanie Balor ${ }^{3}$, Anais Bouissou ${ }^{4}$, Amsha Proag $^{4}$, Raphaël

4 Voituriez ${ }^{5}$, Isabelle Maridonneau-Parini ${ }^{4}$, Wolfgang Baumeister ${ }^{1}$, Serge Dmitrieff ${ }^{2, *}$, Renaud

5 Poincloux ${ }^{4, *}$

6

$7{ }^{1}$ Department of Molecular Structural Biology, Max Planck Institute of Biochemistry, Martinsried,

8 Germany

92 Université de Paris, CNRS, Institut Jacques Monod, Paris, France

$10{ }^{3}$ Plateforme de Microscopie Électronique Intégrative, Centre de Biologie Intégrative, CNRS,

11 Toulouse, France

$12{ }^{4}$ Institut de Pharmacologie et de Biologie Structurale, Université de Toulouse, CNRS, UPS,

13 France

$14{ }^{5}$ Laboratoire Jean Perrin, CNRS, Sorbonne Université, Paris, France

15 * Correspondence: jasnin@biochem.mpg.de; serge.dmitrieff@ijm.fr; renaud.poincloux@ipbs.fr 


\section{Abstract}

17 Actin filaments assemble into force-generating systems involved in diverse cellular functions,

18 including cell motility, adhesion, contractility and division. It remains unclear how networks of actin

19 filaments, which individually generate piconewton forces, can produce forces reaching tens of

20 nanonewtons. Here we use in situ cryo-electron tomography to unveil how the nanoscale

21 architecture of macrophage podosomes enables basal membrane protrusion. We show that the

22 sum of the actin polymerization forces at the membrane is not sufficient to explain podosome

23 protrusive forces. Quantitative analysis of podosome organization demonstrates that the core is

24 composed of a dense network of bent actin filaments storing elastic energy. Theoretical modelling

25 of the network as a spring-loaded elastic material reveals that it exerts forces of up to tens of

26 nanonewtons, similar to those evaluated experimentally. Thus, taking into account not only the

27 interface with the membrane but also the bulk of the network, is crucial to understand force

28 generation by actin machineries. Our integrative approach sheds light on the elastic behavior of

29 dense actin networks and opens new avenues to understand force production inside cells.

\section{Introduction}

32 Actin, one of the most abundant proteins in eukaryotic cells, organize into force-generating

33 filamentous networks, which play pivotal roles in cell motility, adhesion, endocytosis and vesicular

34 traffic ${ }^{1,2}$. Thermodynamics showed that actin polymerization generates mechanical forces by the

35 addition of new monomers at the end of a fluctuating filament ${ }^{3,4}$. Typical stall forces of a 36 polymerizing actin filament were estimated in the 1-10 pN range ${ }^{3,5,6}$, in agreement with

37 experimental values of $1.5 \mathrm{pN}$ found by optical trap measurements ${ }^{7}$. Thus, polymerization of actin

38 filaments against a membrane is capable of extruding thin membrane tubes or forming plasma

39 membrane invaginations in mammalian cells in a force range of a few tens of $\mathrm{pN}$ 8-10.

40 The forces exerted by actin filaments can also reach a much higher regime, in the 41 nanonewton $(\mathrm{nN})$ range ${ }^{11,12}$. At the leading edge of motile cells, branched actin networks in 42 lamellipodial protrusions produce local forces of $\sim 1 \mathrm{nN}{ }^{13,14}$. During yeast endocytosis, the actin 43 machinery generates similar forces to overcome the turgor pressure pushing the invagination 44 outwards ${ }^{15}$. At the basal membrane of myeloid cells, podosomes probe the stiffness of the 45 extracellular environment through the generation of forces reaching tens of $\mathrm{nN}^{16}$. Unlike the lower 46 force regime, the mechanisms by which meshworks of actin filaments produce $\mathrm{nN}$ forces remain 47 unknown.

48 If the force generated by an actin network corresponds to the sum of the polymerization 
forces generated by single filaments pushing against a load, then hundreds to thousands of filaments would need to continuously grow against the surface to reach the $\mathrm{nN}$ range. Alternatively, filaments could push on the membrane with shallow angles ${ }^{17,18}$. It was also proposed that the actin network could store elastic energy, and thus exert a restoring force. In the low force regime, bending of endocytic filaments observed in animal cells has been proposed to store elastic energy for pit internalization ${ }^{19}$. In the large force regime, a theoretical model with non-deformable filaments showed that a large force would cause elastic energy to be stored in cross-linkers deformation ${ }^{20}$. However, none of these hypotheses have been explored in native actin machineries generating forces of several $\mathrm{nN}$, due to the difficulty to combine direct observation of the actin network architecture and the knowledge of the exerted force.

To date, cryo-electron tomography (cryo-ET) is the only technique that resolves single actin filaments inside unperturbed cellular environments ${ }^{21-23}$. Here we use cryo-ET to unveil the three-dimensional (3D) architecture of human macrophage podosomes and elucidate their force generation mechanism. These submicrometric structures are composed of a protrusive core of actin filaments surrounded by an adhesion ring. The balance of forces requires the protrusion force applied by the core on the substrate to be counteracted by a force of equal magnitude. Protrusion force microscopy (PFM) revealed that this balance of forces takes place locally through traction at the adhesion ring $16,24,25$, which has been proposed to be transmitted by radial actin cables connecting the core to the ring ${ }^{25}$. Here we visualize these radial filaments and quantitatively analyze the 3D organization of the core and ring networks. We show that the protrusive forces generated by podosomes cannot be explained by the sum of the actin polymerization forces at the core membrane but by the storage of elastic energy into the dense network of bent actin filaments. These results explain how cellular actin networks can act as a spring-loaded elastic material to exert forces of up to tens of nanonewtons.

\section{Results}

\section{Cryo-ET allows quantitative analysis of filament organization in podosomes}

76 Owing to their size and location at the basal cell membrane, native podosomes are amenable to 77 cryo-ET exploration using cryo-focused ion beam (cryo-FIB) milling sample preparation. We 78 prepared thin vitrified sections (so-called wedges) containing podosomes using shallow incidence 79 angles of the ion beam and subjected them to cryo-ET (Figure 1A and Movie S1). Segmentation

80 of the tomograms revealed that podosomes are made of a core of oblique filaments surrounded 81 by radial filaments (Figure $1 \mathrm{~B}-\mathrm{C}$ and Movie S1), as predicted previously ${ }^{25}$. Other cytoskeletal 
82 elements, cellular organelles, ribosomes and glycogen granules are excluded from the core and

83 radial actin networks, gathering either at the periphery or on top of podosomes (Figure $1 \mathrm{~A}-\mathrm{B}$ and

84 Movie S1).

85 Quantitative analysis of filament organization highlighted the specificity of the actin core 86 in terms of filament length, density and orientation with respect to the basal membrane (Methods 87 and Figures S1-S3). All of these parameters exhibit a sharp transition as a function of the radial 88 distance from the core center (Figure 1D-F). Density is higher inside the core by a factor of 2 to 3 89 (Figure 1D) and filaments display a mean orientation of $50 \pm 21^{\circ}$ relative to the plasma membrane, 90 as compared to the flatter $23 \pm 21^{\circ}$ outside the core (Figure $1 \mathrm{E}$ and Figure S4A-B). Core filaments 91 are shorter than the surrounding radial filaments, with mean lengths of $119 \pm 52 \mathrm{~nm}$ and $181 \pm 134$ $92 \mathrm{~nm}$, respectively (Figure 1F and Figure S4C-D). Further analysis of the transition curves for the 93 filament orientation provided a mean core radius of $203 \pm 38 \mathrm{~nm}$ for a total of ten podosomes 94 (Figure 1E, inset). This agrees with the values obtained from the fits of the other parameters 95 (Figure S5).

Since the milling procedure removed the top of the podosomes, we also imaged podosomes exposed by cell unroofing prior to cryo-fixation to get a complete picture of podosome organization (Figure S6 and Movie S2). In addition to the core and radial filaments observed previously, we detected horizontal filaments on top of the core as well as in between neighboring cores. These filaments, which may have been lifted up from the plasma membrane during podosome growth, could also participate in the generation of the traction forces that counterbalance the protrusion forces generated by podosomes ${ }^{25}$.

104 The sum of the actin polymerization forces at the core membrane is below $\mathbf{1} \mathbf{~ n N}$

105 To evaluate the forces generated by actin polymerization on the plasma membrane beneath the 106 podosome core, where protrusion occurs ${ }^{16,24,25}$, we identified filament segments in the close 107 vicinity of and protruding against the plasma membrane (Figure 2A and Movie S3). We found an 108 average of $45 \pm 29$ filaments per podosome, with a mean orientation of $61 \pm 6^{\circ}$ relative to the 109 plasma membrane (Figure 2B). This rules out the first two hypotheses, namely that 1) hundreds 110 to thousands of filaments are growing at the same time onto the plasma membrane, or that 2) 111 filaments are pushing with shallow angles. In addition, using the upper limit of $10 \mathrm{pN}$ for the stall 112 force of a single filament growing perpendicularly to the membrane ${ }^{3,5,6,26}$, taking into account 113 filament orientation ${ }^{17}$, and considering that all these filaments are polymerizing concomitantly, 114 we evaluated a maximal polymerization force of $615 \pm 396 \mathrm{pN}$ per podosome (ten podosomes 
115 evaluated; Methods and Figure 2C-D). This is one order of magnitude lower than the experimental

116 values ${ }^{16}$. We therefore concluded that the total force produced by polymerization of actin

117 filaments against the plasma membrane is not sufficient to generate the protrusive forces exerted

118 by podosomes.

\section{The actin core stores elastic energy}

121 Next, we tested the third hypothesis, that is, the storage of elastic energy in the network. Visual 122 inspection of the networks indicated that podosome filaments are bent (Figure 3A and Movie S4).

123 The projection of each filament on the vertical plane passing through its two ends highlighted the 124 variation of their local curvature along the filament length (Methods and Figure 3B-C). Quantitative 125 analysis of filament curvature revealed an average compressive strain of $4.2 \pm 0.4 \%$ for all 126 filaments, independently of their localization within the podosome (Figure 3D and Figure S7). We 127 therefore evaluated the elastic energy using the theory of linear elasticity: actin filaments can be 128 modeled as semi-flexible polymers with the following elastic energy at the single filament level ${ }^{27}$ :

$129 \quad u_{\text {elastic }}=\frac{\kappa}{2} \int\left\|\frac{\partial t(s)}{\partial s}\right\|^{2} d s$

130 where $\kappa \sim 4^{*} 10^{-26} \mathrm{~N} \cdot \mathrm{m}^{2}$ is the bending modulus ${ }^{28}, t(s)$ is the tangent vector as a function of the 131 arc-length coordinate $s$, and the integrand represents the square of the local curvature along the 132 filament (Methods and Figure S8A). We evaluated the elastic energy per unit volume as the sum 133 of the energies over all filaments in a given interval at a radial distance $r$ from the core (Figure 134 S8B). The density of elastic energy stored inside the core is much larger than that outside the 135 core, by factors ranging from 3 to 10 (Figure 3E). This is consistent with the larger actin density 136 by factors of 2 to 6 measured inside the core (Figure 1D). Therefore, this specific architecture 137 allows the system to store elastic energy inside the podosome core, ranging from $\sim 10^{4} k_{B} T$ to $138 \sim 5.10^{4} k_{B} T$. This corresponds to $40 k_{B} T$ per filament on average, which is much larger than the 139 scale of thermal fluctuations.

\section{The actin core generates an elastic force in the $\mathrm{nN}$ range}

142 To test whether the stored elastic energy can account for podosome protrusion forces, we next 143 evaluated the elastic force generated through the compression of the actin network. Assuming 144 that the podosome core behaves as a homogeneous elastic material, the force exerted by the 145 core is such that its work for a small deformation of amplitude $\delta h_{\text {core }}$ is equal to $U_{\text {elastic }}^{\text {core }}$ (the total 146 elastic energy stored in the podosome core), with $\delta h_{\text {core }}=\epsilon_{\text {core }} \times h_{\text {core }}\left(\varepsilon_{\text {core }}\right.$ is the average 
147 filament compressive strain in the core and $h_{\text {core }}$ is the core height; Methods). We thus find:

$148 \quad F_{\text {elastic }}^{\text {core }} \approx \frac{U_{\text {elastic }}^{\text {core }}}{\epsilon_{\text {core }} \times h_{\text {core }}}$

149 Using Eq. 2, we found an average elastic force of $9.7 \pm 4.2 \mathrm{nN}$, close to the mean value of $10.4 \pm 3.8$

$150 \mathrm{nN}$ reported from PFM measurements ${ }^{16}$. The elastic force varies significantly between

151 podosomes, with values ranging from 4.1 to $14.4 \mathrm{nN}$ (Figure 4A). This can be explained by the

152 disparity in the core size in our tomograms. Consistently with this, the force per unit area of the

153 core exhibits less variation with an average value of $P=71.1 \pm 12.9 \mathrm{kPa}$ (Methods). This is in

154 agreement, within the experimental margin of error, with the pressure estimated by PFM 16

155 (Methods and Figure 4B) and in the same order of magnitude as that produced during endocytosis

156 in yeast ${ }^{18}$. Note that the elastic energy stored by the network, and thus the elastic force, are

157 underestimated here since we do not take into account the elastic energy stored by crosslinkers

$158 \quad 20$.

\section{Mechanical properties of the podosome}

161 Knowing the elastic force, and thus the pressure $P$, allowed us to estimate the Young's modulus of

162 the core: $Y=P / \epsilon_{\text {core }}=1.7 \mathrm{MPa}$. While this value is several orders of magnitude larger than

163 reported values for reconstituted and cellular actin networks ${ }^{29,30}$, it is compatible with the very

164 high actin density in the core. Actin itself has a Young's modulus $Y_{a}=2.3 \mathrm{GPa}{ }^{31}$. The elastic

165 modulus that can be reached by an actin network can be estimated as $Y=Y_{a} \phi^{2}$, with $\phi$ the

166 volume fraction occupied by actin filaments ${ }^{32}$, yielding an upper limit of $12 \mathrm{MPa}$ in podosomes.

167 Thus, the Young's modulus we find for the podosome core is well within expected values and

168 helps understand how a compressive strain of less than $5 \%$ translates into forces in the 169 nanonewton range.

170 The protrusive force exerted by the core on the substrate is balanced by an opposing force

171 transmitted by the radial filaments to the adhesion ring, which prevents the core from relaxing

172 towards the cell interior. We can estimate the surface tension, $\sigma$, of the 2D meshwork of radial

173 filaments that is required to balance the force $F_{\text {elastic }}^{\text {core }}$, knowing the mean radius of the core,

$174 r_{c o r e}$, and the mean angle of the radial filaments, $\theta_{\text {radial }}$, with respect to the membrane plane

175 (Figure 4C and Figure S4B):

$176 \sigma=\frac{F_{\text {elastic }}^{\text {core }}}{2 \pi r_{\text {core }} \sin \theta_{\text {radial }}} \sim 20 \mathrm{mN} / \mathrm{m}$

177 While this tension is one order of magnitude larger than the cortical tension of rounding mitotic 
178 cells ${ }^{33}$, it is the same order of magnitude as the tension inferred for some adhering cells, from 6

$179 \mathrm{mN} / \mathrm{m}^{34}$ to $100 \mathrm{mN} / \mathrm{m}^{35}$.

\section{Discussion}

182 In summary, our results showed that mechanical energy accumulates inside the podosome core 183 through bending of the dense network of actin filaments (Figure 1 and Figure 3). To relax, the 184 elastic energy produces an elastic force, which is balanced by radial tension between the actin core and the adhesion ring. Indeed, we found that the elastic force computed from the bending energy matches the protrusive force measured experimentally (Figure 4). We revealed that the load borne by actin filaments in contact with the membrane is greater than the stall force, making their growth thermodynamically unfavorable (Figure 2). In addition, the need to synchronize their growth against the membrane would drastically slow down the polymerization process ${ }^{36}$. Thus, how the network assembles under force remains to be understood.

One possibility is that actin filaments do not grow directly against the load at the membrane, which would instead be borne by the existing dense and compressed network. They would rather grow bent in the bulk of the podosome core in a dense environment, thus increasing the elastic energy stored by the network, which can then relax by pushing against the membrane. An estimation of the energy released by actin polymerization in the core, by summing over all actin monomers ${ }^{17}$, yields $1-3.10^{5} k_{B} T$, which is one order of magnitude larger than the elastic energy $U_{\text {elastic }}^{\text {core }}$. While some of the polymerization energy will be dissipated as heat, the polymerization reaction can still significantly contribute to the loading of the network.

Another possibility is that the filaments grow under a much smaller force, and that the network is then loaded by the active tension of the actomyosin cables driven by myosin II. This would be consistent with the decrease in pushing force when myosin II is inhibited ${ }^{24}$. In this scenario, the energy source would be mostly ATP consumed by myosin II rather than the polymerization energy. We expect that a combination of experiments and simulation will be able to clarify the assembly of such a spring-loaded network.

In conclusion, to understand the forces applied by actin assemblies, it is not sufficient to 207 account. Through a combination of quantitative analysis and modelling of the native 3D 208 architecture of macrophage podosomes, we showed that elastic energy is stored in actin networks 209 in vivo, allowing forces of the order of ten $\mathrm{nN}$ to be produced. These results highlight the 210 possibilities opened by exploring the architecture of actin networks in situ at the molecular scale. 
bioRxiv preprint doi: https://doi.org/10.1101/2021.04.13.439622; this version posted April 14, 2021. The copyright holder for this preprint (which

was not certified by peer review) is the author/funder, who has granted bioRxiv a license to display the preprint in perpetuity. It is made available under aCC-BY-NC-ND 4.0 International license.

211 Given the rapid progress of cryo-ET, we expect that, building on our biophysical approach, it will

212 soon be possible to address other degrees of freedom for elastic energy storage, such as

213 crosslinker deformation and filament twisting, and to shed light on other modes of force generation

214 by cellular machineries. 


\section{Methods}

\section{Differentiation and culture of primary monocyte-derived macrophages}

217 Human monocytes were isolated from the blood of healthy donors as described previously ${ }^{37}$. 218 Cells were resuspended in cold phosphate buffered saline (PBS) supplemented with 2 mM EDTA, $2190.5 \%$ heat-inactivated Fetal Calf Serum (FCS) at pH 7.4 and magnetically sorted with magnetic 220 microbeads coated with antibodies directed against CD14 (Miltenyi Biotec). Monocytes were then 221 seeded on glass coverslips at $1.5 \times 10^{6}$ cells/well in six-well plates in RPMI 1640 (Invitrogen) 222 without FCS. After $2 \mathrm{~h}$ at $37^{\circ} \mathrm{C}$ in a humidified $5 \% \mathrm{CO}_{2}$ atmosphere, the medium was replaced by 223 RPMI containing 10\% FCS and $20 \mathrm{ng} / \mathrm{mL}$ of Macrophage Colony-Stimulating Factor (M-CSF) 224 (Peprotech). For experiments, cells were harvested at day 7 using trypsin-EDTA (Fisher 225 Scientific) and centrifugation (320g, $10 \mathrm{~min})$.

\section{Cell vitrification}

228 Gold EM grids with R1/4 holey $\mathrm{S}_{\mathrm{i}} \mathrm{O}_{2}$ film (Quantifoil) were glow-discharged in a EasiGlow (Pelco) 229 glow discharge system. After grid sterilization under UV light, the cell suspension containing 230 fiducials was seeded onto the grids and incubated for $2 \mathrm{~h}$ at $37^{\circ} \mathrm{C}$ to let the cells adhere to the 231 grids, resulting in 3 to 4 cells per grid square. For cell vitrification, grids were loaded into the 232 thermostatic chamber of a Leica EM-GP automatic plunge freezer, set at $20^{\circ} \mathrm{C}$ and $95 \%$ humidity.

233 Excess solution was blotted away for $10 \mathrm{~s}$ with a Whatman filter paper $\mathrm{n}^{\circ} 1$, and the grids were 234 immediately flash frozen in liquid ethane cooled at $-185^{\circ} \mathrm{C}$.

\section{Unroofing}

237 When indicated, macrophages plated on grids were unroofed prior to vitrification. Cells were 238 unroofed using distilled water containing cOmplete ${ }^{\mathrm{TM}}$ protease inhibitors (Roche) and $10 \mu \mathrm{g} / \mathrm{mL}$ 239 phalloidin (Sigma-Aldrich P2141) for $30 \mathrm{~s}$.

\section{Cryo-FIB milling}

242 Plunge-frozen EM grids were clipped into Autogrid frames modified for wedge milling under 243 shallow angles ${ }^{38}$. Autogrids were mounted into a custom-built FIB-shuttle and transferred using 244 a cryo-transfer system (PP3000T, Quorum) to the cryo-stage of a dual-beam Quanta 3D FIB/SEM 245 (Thermo Fisher Scientific) operated at liquid nitrogen temperature ${ }^{39}$. The support film close to the 
246 cells of interest was sputtered away with high beam currents of 0.5-1.0 nA to provide a reference

247 in Z direction for wedge milling. Cells were first milled roughly at very shallow angles (typically 2-

$2485^{\circ}$ of the incident ion beam) with beam currents of 300-500 pA. Advancement of the milling was

249 monitored by SEM at $5 \mathrm{kV}$ and $5.92 \mathrm{pA}$. Closer to the cell surface, beam currents of 50-100 pA

250 were used for fine milling. Once all the wedges were prepared on the grid, a final polishing step

251 at 30-50 pA was performed to limit surface contamination.

252

\section{Cryo-ET and tomogram reconstruction}

254 Wedges were loaded vertically to the tilt axis in a Titan Krios transmission electron microscope 255 (Thermo Fisher Scientific) equipped with a $300 \mathrm{kV}$ field-emission gun, Volta phase plates (VPPs) 256 (Danev et al., 2014), a post-column energy filter (Gatan, Pleasanton, CA, USA) and a 4k x 4k K2 257 Summit direct electron detector (Gatan) operated with SerialEM. The VPPs were aligned and 258 used as described previously ${ }^{40}$. Low-magnification images were recorded at 2250x. High259 magnification tilt-series were recorded at 33,000x (calibrated pixel size $0.421 \mathrm{~nm}$ ) with a target 260 defocus for phase-plate imaging of $0 \mu \mathrm{m}$. Bi-directional tilt series were acquired typically from $26130^{\circ}$ to $+60^{\circ}$ and $-32^{\circ}$ to $-60^{\circ}$ with a tilt increment of $2^{\circ}$ and a total dose between 150 and 200 e$262 / \AA^{2}$. Frames were aligned with in-house software (K2Align) based on procedures developed by $\mathrm{Li}$ 263 et al. ${ }^{41}$. Tilt series were aligned using the gold beads deposited on the surface of the support film 264 as fiducial markers. 3D reconstructions with final pixel sizes of $1.684 \mathrm{~nm}$ were obtained by 265 weighted-back projection using the IMOD software ${ }^{42}$.

\section{Automated filament segmentation}

268 The $4 \mathrm{x}$ binned tomograms (pixel size of $1.684 \mathrm{~nm}$ ) were subjected to nonlocal-means filtering 269 using the Amira software ${ }^{43}$ provided by Thermo Fisher Scientific. Actin filaments were traced 270 using an automated segmentation algorithm based on a generic filament as a template ${ }^{44}$, with a 271 diameter of $8 \mathrm{~nm}$ and a length of $42 \mathrm{~nm}$. To reduce background noise, short filamentous structures 272 with lengths below $60 \mathrm{~nm}$ (or $50 \mathrm{~nm}$ for the unroofed podosomes) were filtered out.

273 The coordinates of the segmented filaments were exported from Amira and used as input for data 274 analysis in MATLAB (The MathWorks) and in python (https://gitlab.com/jhervy/podosome-demo).

275 The coordinates of the filaments were resampled every $3 \mathrm{~nm}$ to give the same weight to every 276 point along a filament ${ }^{21}$. 
All the parameters (filament length, density and orientation, compressive strain and density of elastic energy) were evaluated as detailed in Figures S1-S3, S7 and S8, respectively. The plots as a function of the radial distance were computed by considering all the values in a given interval $[r, r+\delta]$ at a radial distance $r$ from the core center. A list of 21 linearly-spaced values starting from 0 to $500 \mathrm{~nm}$ was used for the binning of the radial distance, which corresponds to an interval

284 value of $\delta=25 \mathrm{~nm}$ (Figure 1D-F, Figure 3D-E, Figure S5A).

\section{Estimation of the core radius}

287 All the parameters except the compressive strain were fitted as a function of the radial distance $r$ 288 from the core center using the following equation:

$m(r)=\frac{1}{2}\left[(a+b)+(b-a) \tanh \left(\frac{r-r_{0}}{r_{s}}\right)\right]$

$290 m(r)$ is either a decreasing function (for the filament density, filament orientation and the density 291 of elastic energy) or an increasing function (for the filament length) with two saturation values set 292 by the parameters $a$ and $b$ (Figure 1D-F and Figure 3E). The parameter $r_{0}$ corresponds to the 293 radial distance for which the slope of the tangent line is maximum; the parameter $r_{s}$ defines the 294 transition range between the two saturating values (Figure S5A). We used the parameter $r_{0}$ from 295 the fit of the orientation as a measure of the core radius (Figure S5B).

\section{Polymerization force}

298 To estimate the polymerization force generated at the core membrane, the protrusive filaments, 299 i.e. those in the immediate vicinity of the plasma membrane, were considered. Specifically, all the 300 filament portions up to $10 \mathrm{~nm}$ away from the membrane were taken into account (Figure 2A). A 301 single filament growing perpendicularly to the membrane generates a force given by 4 :

$302 f_{a}=\frac{k_{B} T}{\delta} \ln \left(\frac{C}{C_{c}}\right)$

303 where $k_{B} T=4.1110^{-21} \mathrm{~J}, \delta=2.75 \mathrm{~nm}$ is the displacement induced by the addition of one actin 304 monomer, $C$ is the concentration of actin monomers in solution and $C_{C}$ is the critical concentration. 305 Using the values $C=150 \mu \mathrm{M}$ as estimated in ${ }^{45}$ and $C_{c}=0.06 \mu \mathrm{M}$ for the critical concentration at 306 the plus end as measured in vitro ${ }^{46,47}$, we found an upper limit of $11 \mathrm{pN}$ for the stall force. Thus, 307 the factor $\ln \left(C / C_{c}\right)$ was set to 7 leading to a stall force of $10 \mathrm{pN}$ for a single filament growing 308 perpendicularly to the membrane. This value was increased by a factor $1 / \sin (\theta)$ for a filament 309 having an orientation $\theta$ relative to the membrane ${ }^{17}$ (Figure 2C). Therefore, the total 
310 polymerization force was computed as follows:

$311 \quad F_{\text {polym }}^{\text {core }}=f_{a} \sum_{i=1}^{n_{p}} \frac{1}{\sin \left(\theta_{i}\right)}$

312 where $n_{p}$ is the number of protrusive filaments and $\theta_{i}$ is the average orientation for the protrusive

313 filament $i$ (Figure 2D).

\section{Local curvature}

315 The local curvature was evaluated between two consecutive points using their respective tangent 316 vector $t$ as follows:

$317 \quad \gamma_{i, i+1}=\frac{1}{a} \arccos \left(t_{i} \cdot t_{i+1}\right)$

318 where $a$ is their relative distance set to $3 \mathrm{~nm}$ in our segmentation procedure.

\section{Local elastic energy}

320 The local energy is proportional to the square of the local curvature and can be discretized as 321 follows:

$322 \quad u_{i, i+1}^{\text {elastic }}=\frac{\kappa}{2}\left(\gamma_{i, i+1}\right)^{2}$

323 where $\kappa$ is the bending modulus ${ }^{28}$.

\section{Total elastic energy}

326 The total elastic energy inside the core was computed by summing the local energy over all the

327 filament points that are within the radial distance domain $r \in\left[0, r_{\text {core }}\right]$, where $r_{\text {core }}$ is the core 328 radius (Figure $\mathrm{S} 8$ ).

\section{Elastic force}

330 The elastic force pushing perpendicularly to the membrane is the derivative of the core elastic 331 energy with respect to the height $h_{\text {core }}$ of the core:

$332 \quad F_{\text {elastic }}^{\text {core }}=\frac{d U_{\text {elastic }}^{\text {core }}}{d h_{\text {core }}} \approx \frac{\delta U_{\text {elastic }}^{\text {core }}}{\delta h_{\text {core }}}$

333 with $\delta$ indicating small changes. Indeed, in the core, the average compressive strain of the 334 filaments is $\epsilon_{\text {core }} \sim 0.04$ (Figure 3D) and therefore considered to be small. Assuming all elastic 335 energy to be released in the resting state, we have $\delta U_{\text {elastic }}^{\text {core }}=U_{\text {elastic }}^{\text {core }}$ and $\delta h_{\text {core }}=\epsilon_{\text {core }} \times h_{\text {core }}$. 
336 Therefore, we find:

$337 \quad F_{\text {elastic }}^{\text {core }} \approx \frac{U_{\text {elastic }}^{\text {core }}}{\epsilon_{\text {core }} \times h_{\text {core }}}$

338 Note that this is an order of magnitude estimate: since the actin network is assembled under

339 pressure, it is likely that its resting state has a non-zero elastic energy, in which case the force is

340 overestimated. The precise architecture of the network could also yield a prefactor in the relation

341 between $\delta h_{\text {core }}$ and $\epsilon_{\text {core }}$. Lastly, the elastic energy could be underestimated: part of it might be

342 stored in crosslinker elasticity and in tension of the non-bent segments of actin filaments.

344 Pressure induced in the podosome core

345 Assuming a circular shape of radius $r_{\text {core }}$ for the core, the pressure was computed as:

$346 P=\frac{F_{\text {elastic }}^{\text {core }}}{\pi r_{\text {core }}^{2}}$

347 In the rest of this section, the values used to compute the pressure from the cryo-ET data and the 348 PFM force measurements reported in ${ }^{16}$ are detailed.

\section{a) From the tomograms}

351 The pressure induced by the compression of the actin filaments (labelled as "Elastic", Figure 4A)

352 and by the polymerization at the membrane (labelled as "Polymerization") were evaluated using 353 the values for $r_{\text {core }}$ estimated from the fits of the orientation data (Figure 1E, inset).

\section{b) From PFM force measurements}

356 The pressure $P_{P F M}$ was computed using the force value $F=10.4 \pm 3.8 \mathrm{nN}$ reported in ${ }^{16}$ and

$357 \quad r_{\text {core }}=203.1 \pm 37.8 \mathrm{~nm}$ estimated from the average of 10 podosomes (black dashed line in Figure

$3581 \mathrm{E}$, inset). The error bar for this value was computed using the propagation of uncertainty method 359 as follows:

$360 \Delta P_{P F M}=\frac{2 F_{\text {elastic }}^{\text {core }}}{\pi r_{\text {core }}^{3}} \Delta r_{\text {core }}+\frac{1}{\pi r_{\text {core }}^{2}} \Delta F_{\text {elastic }}^{\text {core }}$

361 We found $P_{P F M}=80.0 \pm 59.0 \mathrm{kPa}$, with the margin of error $\triangle P_{P F M}$ represented as a rectangular 362 blue area filled in Figure 4B.

\section{Resource availability}

365 All data and code used in the analysis are available upon request to the corresponding authors. 


\section{References}

3681 Pollard, T. D. \& Cooper, J. A. Actin, a central player in cell shape and movement. Science 326, 1208-1212, doi:10.1126/science.1175862 (2009).

3702 Harris, A. R., Jreij, P. \& Fletcher, D. A. Mechanotransduction by the Actin Cytoskeleton: Converting Mechanical Stimuli into Biochemical Signals. Annual Review of Biophysics, Vol 47 47, 617-631, doi:10.1146/annurev-biophys-070816-033547 (2018). Mogilner, A. \& Oster, G. Cell motility driven by actin polymerization. Biophysical Jjournal 71, 3030-3045 (1996).

$4 \quad$ Peskin, C. S., Odell, G. M. \& Oster, G. F. Cellular motions and thermal fluctuations: the Brownian ratchet. Biophysical Jjournal 65, 316-324, doi:10.1016/S0006-3495(93)81035X (1993).

$5 \quad$ Hill, T. L. \& Kirschner, M. W. Subunit treadmilling of microtubules or actin in the presence of cellular barriers: possible conversion of chemical free energy into mechanical work. Proceedings of the National Academy of Sciences of the United States of America 79, 490-494, doi:10.1073/pnas.79.2.490 (1982). Hill, T. L. \& Kirschner, M. W. Bioenergetics and kinetics of microtubule and actin filament assembly-disassembly. Int Rev Cytol 78, 1-125 (1982).

Footer, M. J., Kerssemakers, J. W., Theriot, J. A. \& Dogterom, M. Direct measurement of force generation by actin filament polymerization using an optical trap. Proceedings of the National Academy of Sciences of the U.S.A. 104, 2181-2186, doi:10.1073/pnas.0607052104 (2007). Derenyi, I., Julicher, F. \& Prost, J. Formation and interaction of membrane tubes. Physical review letters 88, 238101, doi:10.1103/PhysRevLett.88.238101 (2002).

9 Atilgan, E., Wirtz, D. \& Sun, S. X. Mechanics and dynamics of actin-driven thin membrane protrusions. Biophysical journal 90, 65-76, doi:10.1529/biophysj.105.071480 (2006).

11 Giardini, P. A., Fletcher, D. A. \& Theriot, J. A. Compression forces generated by actin Ding, B. et al. Recording the dynamic endocytosis of single gold nanoparticles by AFMbased force tracing. Nanoscale 7, 7545-7549, doi:10.1039/c5nr01020a (2015). comet tails on lipid vesicles. Proceedings of the National Academy of Sciences of the United States of America 100, 6493-6498, doi:10.1073/pnas.1031670100 (2003).

13 Mueller, J. et al. Load Adaptation of Lamellipodial Actin Networks. Cell 171, 188-200 e116, Parekh, S. H., Chaudhuri, O., Theriot, J. A. \& Fletcher, D. A. Loading history determines the velocity of actin-network growth. Nature cell biology 7, 1219-1223, doi:10.1038/ncb1336 (2005).

14 Prass, M., Jacobson, K., Mogilner, A. \& Radmacher, M. Direct measurement of the lamellipodial protrusive force in a migrating cell. The Journal of cell biology 174, 767-772, doi:10.1083/jcb.200601159 (2006).

15 Basu, R., Munteanu, E. L. \& Chang, F. Role of turgor pressure in endocytosis in fission yeast. Molecular biology of the cell 25, 679-687, doi:10.1091/mbc.E13-10-0618 (2014).

16 Proag, A. et al. Working together: spatial synchrony in the force and actin dynamics of podosome first neighbors. ACS nano 9, 3800-3813, doi:10.1021/nn506745r (2015).

17 Dmitrieff, S. \& Nedelec, F. Amplification of actin polymerization forces. The Journal of cell biology 212, 763-766, doi:10.1083/jcb.201512019 (2016). 
41118 Mund, M. et al. Systematic Nanoscale Analysis of Endocytosis Links Efficient Vesicle

412

413

414

415

416

417

418

419

420

421

422

423

424

425

426

427

428

429

430

431

432

433

434

435

436

437

438

439

440

441

442

443

444

445

446

447

448

449

450

451

452

453

454

455

456

457

Formation to Patterned Actin Nucleation. Cell 174, 884-896 e817, doi:10.1016/j.cell.2018.06.032 (2018).

19 Akamatsu, M. et al. Principles of self-organization and load adaptation by the actin cytoskeleton during clathrin-mediated endocytosis. eLife 9, doi:10.7554/eLife.49840 (2020).

20 Ma, R. \& Berro, J. Structural organization and energy storage in crosslinked actin assemblies. PLoS computational biology 14, e1006150, doi:10.1371/journal.pcbi.1006150 (2018).

21 Jasnin, $M$. et al. Three-dimensional architecture of actin filaments in Listeria monocytogenes comet tails. Proceedings of the National Academy of Sciences of the United States of America 110, 20521-20526, doi:10.1073/pnas.1320155110 (2013).

22 Jasnin, M. et al. The Architecture of Traveling Actin Waves Revealed by Cryo-Electron Tomography. Structure 27, 1211-1223 e1215, doi:10.1016/j.str.2019.05.009 (2019).

23 Jasnin, M., Ecke, M., Baumeister, W. \& Gerisch, G. Actin Organization in Cells Responding to a Perforated Surface, Revealed by Live Imaging and Cryo-Electron Tomography. Structure 24, 1031-1043, doi:10.1016/j.str.2016.05.004 (2016).

24 Labernadie, A. et al. Protrusion force microscopy reveals oscillatory force generation and mechanosensing activity of human macrophage podosomes. Nat. Commun. 5, 5343, doi:10.1038/ncomms6343 (2014).

25 Bouissou, A. et al. Podosome Force Generation Machinery: A Local Balance between Protrusion at the Core and Traction at the Ring. ACS nano 11, 4028-4040, doi:10.1021/acsnano.7b00622 (2017).

26 Hill, T. L. Microfilament or microtubule assembly or disassembly against a force. Proceedings of the National Academy of Sciences of the United States of America 78, 5613-5617, doi:10.1073/pnas.78.9.5613 (1981).

27 Broedersz, C. P. \& MacKintosh, F. C. Modeling semiflexible polymer networks. Rev Mod Phys 86, 995-1036, doi:10.1103/RevModPhys.86.995 (2014).

28 De La Cruz, E. M. \& Gardel, M. L. Actin Mechanics and Fragmentation. Journal of Biological Chemistry 290, 17137-17144, doi:10.1074/jbc.R115.636472 (2015).

29 Planade, J. et al. Mechanical stiffness of reconstituted actin patches correlates tightly with endocytosis efficiency. PLoS biology 17, e3000500, doi:10.1371/journal.pbio.3000500 (2019).

30 Bieling, P. et al. Force Feedback Controls Motor Activity and Mechanical Properties of Self-Assembling Branched Actin Networks. Cell 164, 115-127, doi:10.1016/j.cell.2015.11.057 (2016).

31 Gittes, F., Mickey, B., Nettleton, J. \& Howard, J. Flexural rigidity of microtubules and actin filaments measured from thermal fluctuations in shape. The Journal of cell biology 120, 923-934, doi:10.1083/jcb.120.4.923 (1993).

32 Satcher, R. L., Jr. \& Dewey, C. F., Jr. Theoretical estimates of mechanical properties of the endothelial cell cytoskeleton. Biophysical journal 71, 109-118, doi:10.1016/S00063495(96)79206-8 (1996).

33 Fischer-Friedrich, E., Hyman, A. A., Julicher, F., Muller, D. J. \& Helenius, J. Quantification of surface tension and internal pressure generated by single mitotic cells. Scientific reports 4, doi:Artn 621310.1038/Srep06213 (2014).

34 Paul, R., Heil, P., Spatz, J. P. \& Schwarz, U. S. Propagation of mechanical stress through the actin cytoskeleton toward focal adhesions: Model and experiment. Biophysical journal 
94, 1470-1482, doi:10.1529/biophysj.107.108688 (2008).

35 Manifacier, I. et al. The consequence of substrates of large-scale rigidity on actin network tension in adherent cells. Comput Method Biomec 22, 1073-1082, doi:10.1080/10255842.2019.1629428 (2019).

36 Tsekouras, K., Lacoste, D., Mallick, K. \& Joanny, J. F. Condensation of actin filaments pushing against a barrier. New journal of physics 13, doi:Artn 10303210.1088/13672630/13/10/103032 (2011).

37 Van Goethem, E., Poincloux, R., Gauffre, F., Maridonneau-Parini, I. \& Le Cabec, V. Matrix Architecture Dictates Three-Dimensional Migration Modes of Human Macrophages: Differential Involvement of Proteases and Podosome-Like Structures. Journal of immunology 184, 1049-1061, doi:10.4049/jimmunol.0902223 (2010).

38 Rigort, A. et al. Micromachining tools and correlative approaches for cellular cryo-electron tomography. J. Struct. Biol. 172, 169-179, doi:10.1016/j.jsb.2010.02.011 (2010).

39 Rigort, A. et al. Focused ion beam micromachining of eukaryotic cells for cryoelectron tomography. Proceedings of the National Academy of Sciences of the United States of America 109, 4449-4454, doi:10.1073/pnas.1201333109 (2012).

40 Fukuda, Y., Laugks, U., Lucic, V., Baumeister, W. \& Danev, R. Electron cryotomography of vitrified cells with a Volta phase plate. Journal of structural biology 190, 143-154, doi:10.1016/j.jsb.2015.03.004 (2015).

$41 \mathrm{Li}, \mathrm{X}$. et al. Electron counting and beam-induced motion correction enable near-atomicresolution single-particle cryo-EM. Nature methods 10, 584-590, doi:10.1038/nmeth.2472 (2013).

42 Kremer, J. R., Mastronarde, D. N. \& McIntosh, J. R. Computer visualization of threedimensional image data using IMOD. Journal of structural biology 116, 71-76, doi:10.1006/jsbi.1996.0013 (1996).

43 Stalling, D., Westerhoff, M., Hege H-C Amira: A highly interactive system for visual data analysis. Elsvier, Butterwoth-Heinemann, 749-767 (2005).

44 Rigort, A. et al. Automated segmentation of electron tomograms for a quantitative description of actin filament networks. J. Struct. Biol. 177, 135-144, doi:10.1016/j.jsb.2011.08.012 (2012).

45 Koestler, S. A. et al. F- and G-actin concentrations in lamellipodia of moving cells. PloS one 4, e4810, doi:10.1371/journal.pone.0004810 (2009).

46 Pollard, T. D., Blanchoin, L. \& Mullins, R. D. Molecular mechanisms controlling actin filament dynamics in nonmuscle cells. Annu Rev Biophys Biomol Struct 29, 545-576, doi:10.1146/annurev.biophys.29.1.545 (2000).

47 Le Clainche, C. \& Carlier, M. F. Regulation of actin assembly associated with protrusion and adhesion in cell migration. Physiol Rev88, 489-513, doi:10.1152/physrev.00021.2007 (2008).

\section{Acknowledgements}

This work benefited from the assistance of Vanessa Soldan from the Multiscale Electron Imaging platform (METi) of the Centre de Biologie Intégrative (CBI). The authors thank Martin Lenz for helpful discussions. This work was supported by the Human Frontier Science Program (RGP0035/2016), la Fondation pour la Recherche Médicale (FRM DEQ2016 0334894), a CNRS 
502 Momentum fellowship, l'Agence Nationale de la Recherche and Deutsche

503 Forschungsgemeinschaft (ANR-DFG JA-3038/2-1) and with financial support from ITMO Cancer

504 of Aviesan on funds managed by Inserm.

505

506 Contributions

507 MJ, RP designed the project. AB, RP prepared the cells. SB vitrified the cells. MJ performed cryo-

508 FIB milling, cryo-ET, tomogram reconstruction and segmentation. MJ, JH, AP, RP designed and 509 performed the experimental analysis. RV, SD designed the theoretical analysis. JH, SD performed 510 the theoretical analysis. MJ, SD, RP supervised the project. MJ, IMP, WB, SD, RP obtained 511 funding. MJ, JH, SD, RP wrote the manuscript with input from the others.

512

\section{Ethics declaration}

514 The authors declare no competing interests. 

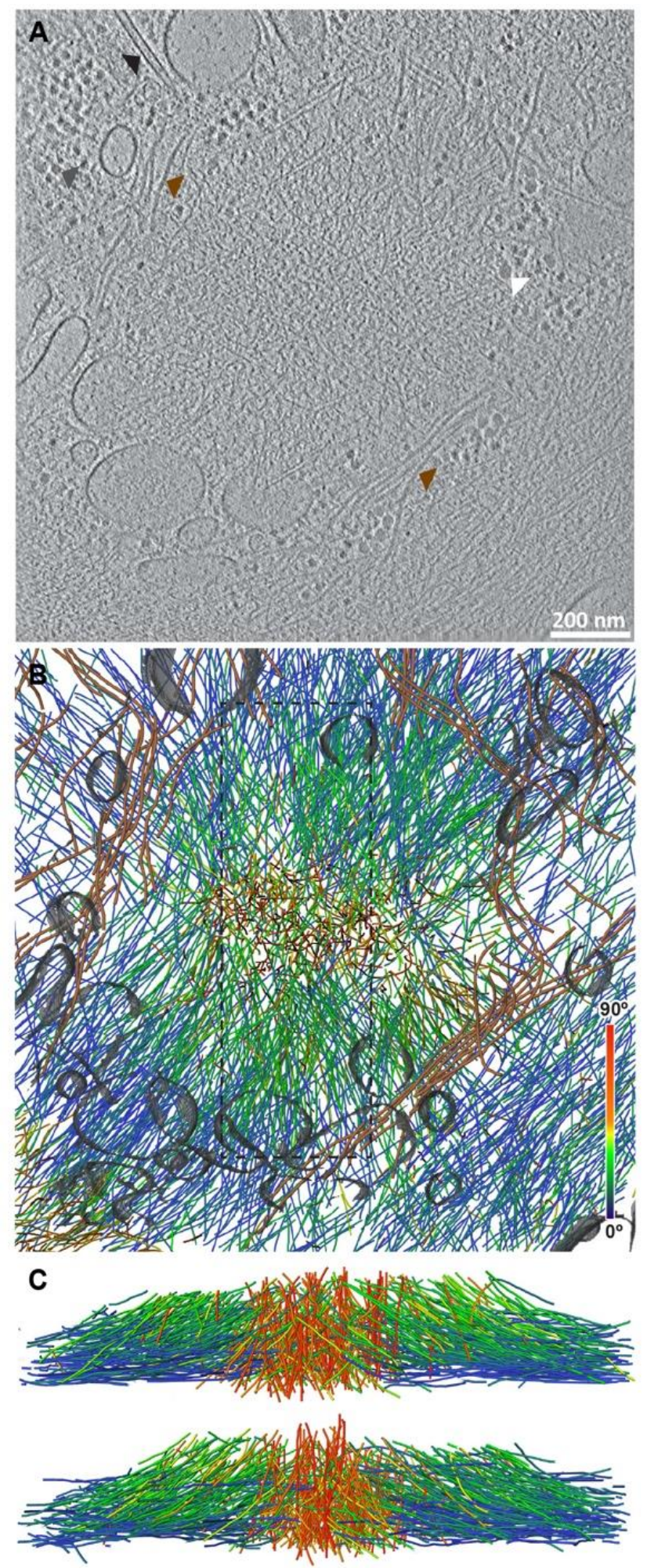

518 Fig. 1: Cryo-electron tomography allows organization in native podosomes.
D

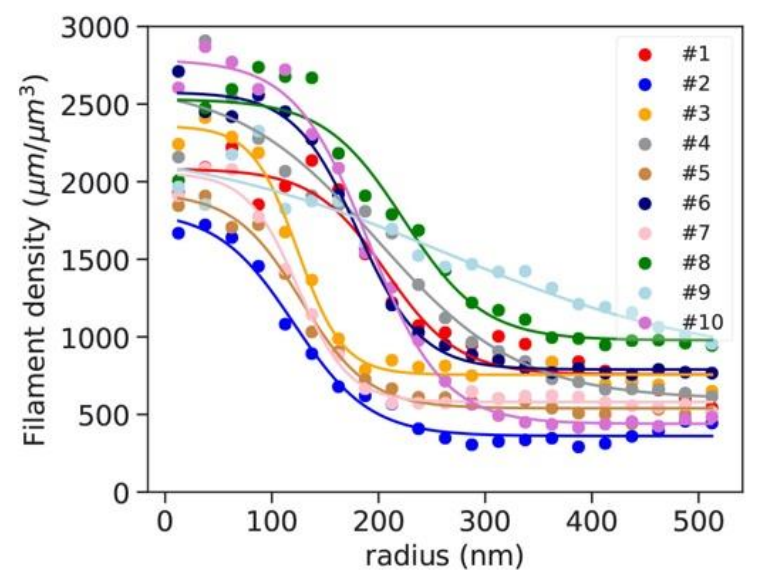

E

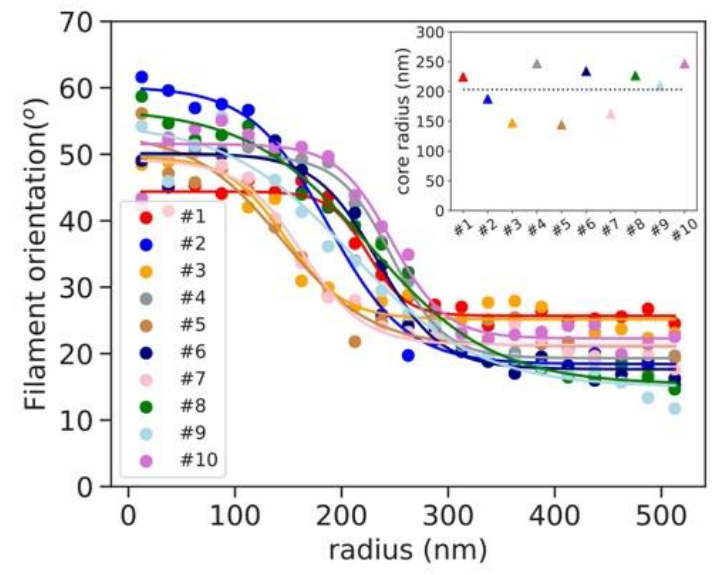

$\mathbf{F}$

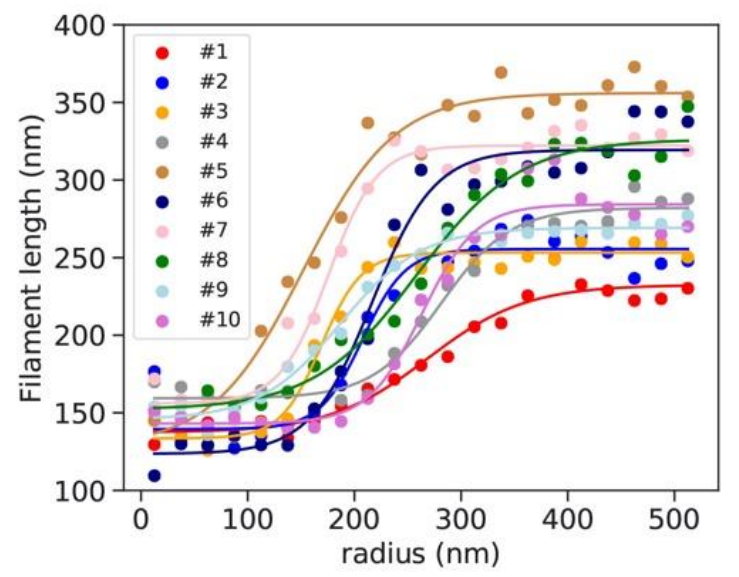

A: Slice from a tomographic volume acquired in a frozen-hydrated human macrophage revealing the podosome environment. Colored arrows point to ribosomes (grey), glycogen granules (white), 
522 a microtubule (black), and intermediate filaments (brown). See also Movie S1.

523 B: Orthographic view of the corresponding 3D segmentation of the actin filaments showing their

524 relative orientation with respect to the basal membrane, intermediate filaments (brown), and

525 organelle membranes (grey).

526 C: Perspective views of the actin filaments in the volume indicated by a dotted rectangle in (B) 527 shown from the left (top) and right (bottom) sides.

528 D-F: Average filament density (D), orientation (E) and length (F) as a function of the radial 529 distance from the core center for ten tomograms. Inset in (E): Corresponding values estimated 530 for the core radius (Methods). The podosome shown in (A-C) corresponds to \#9. 


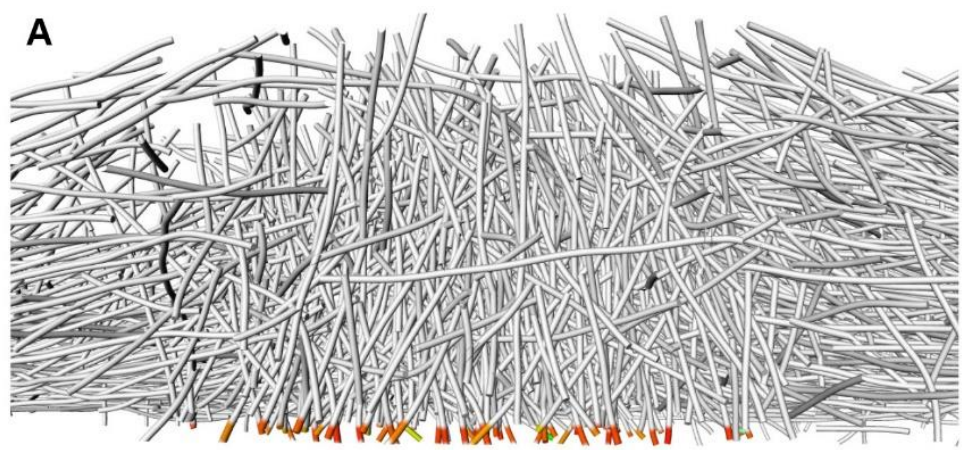

B
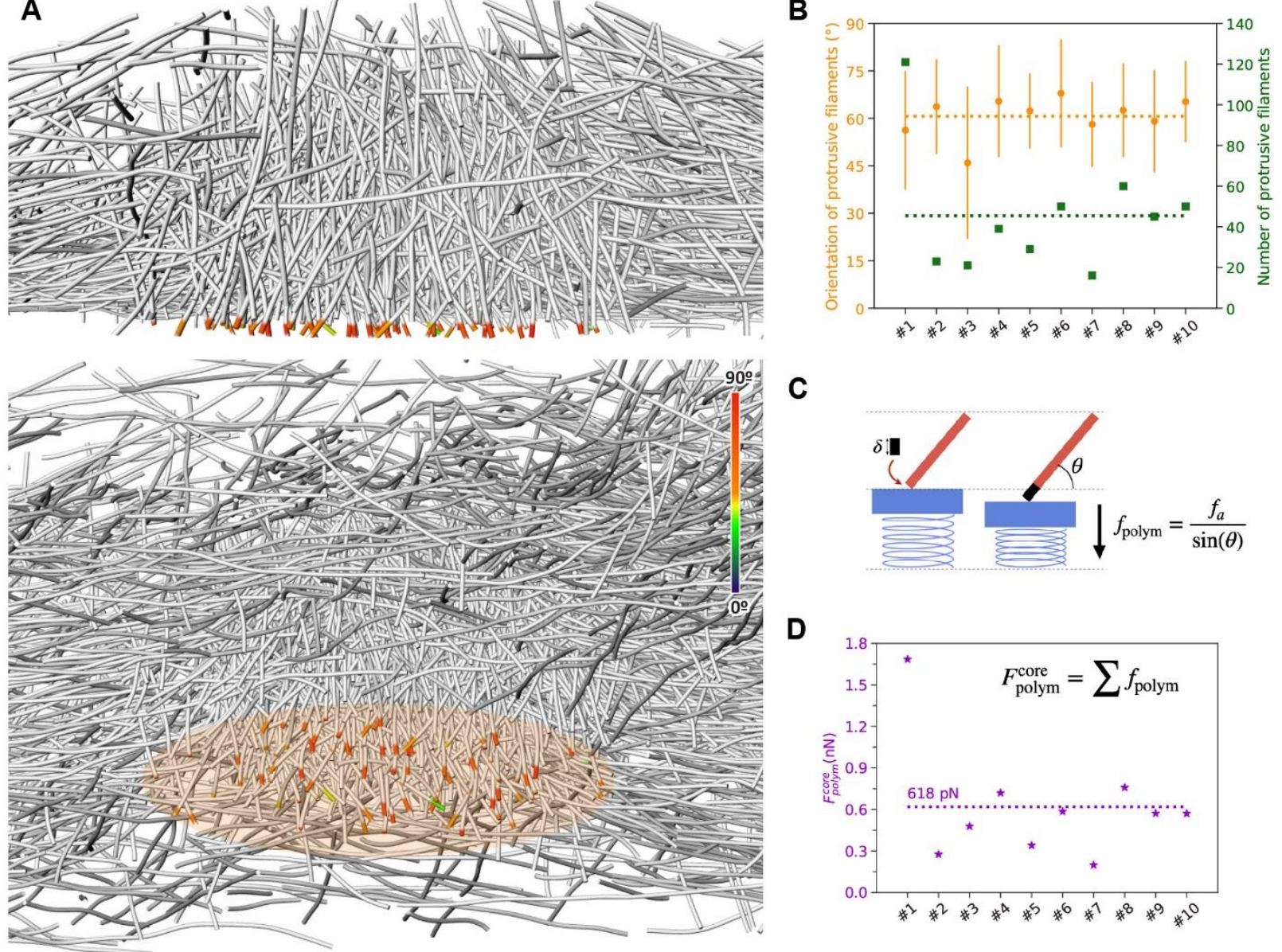

C

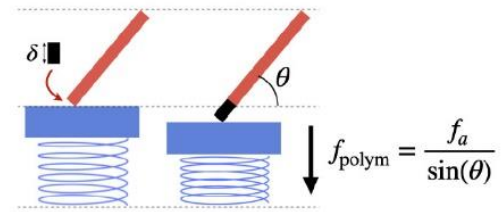

D

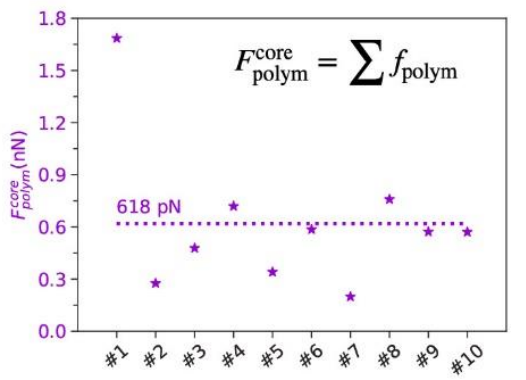

Fig. 2. The sum of the actin polymerization forces at the core membrane is below $1 \mathrm{nN}$.

533 A: (Top) Perspective view of a cross-section through podosome \#8 displaying in color the part of 534 the core filaments pushing against the plasma membrane. (Bottom) Orthographic view of the same podosome from a different angle. The color map corresponds to the filament orientation with respect to the basal membrane. See also Movie S3.

537 B: Mean orientation (orange) and number (green) of polymerizing filaments at the core 538 membrane.

539 C: Scheme representing the polymerization force generated by the addition of a new monomer 540 at the growing end of a filament with an inclination $\theta$ relative to the membrane.

541 D: Estimated polymerization force generated at the core membrane. 


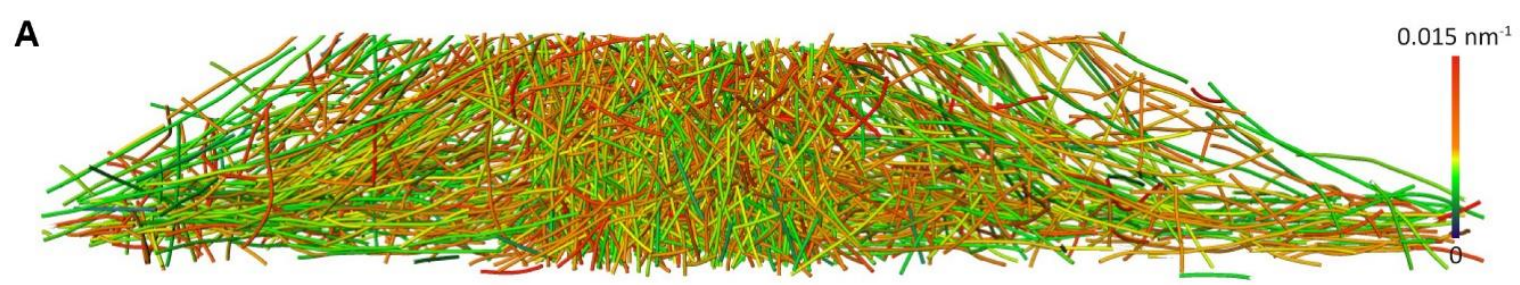

B

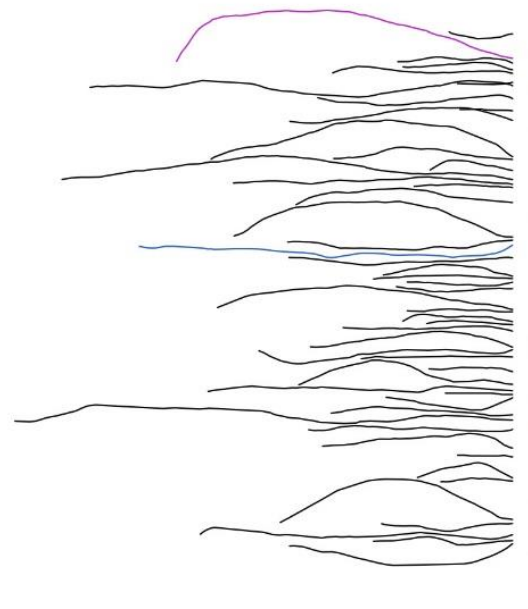

Filaments outside the core

D

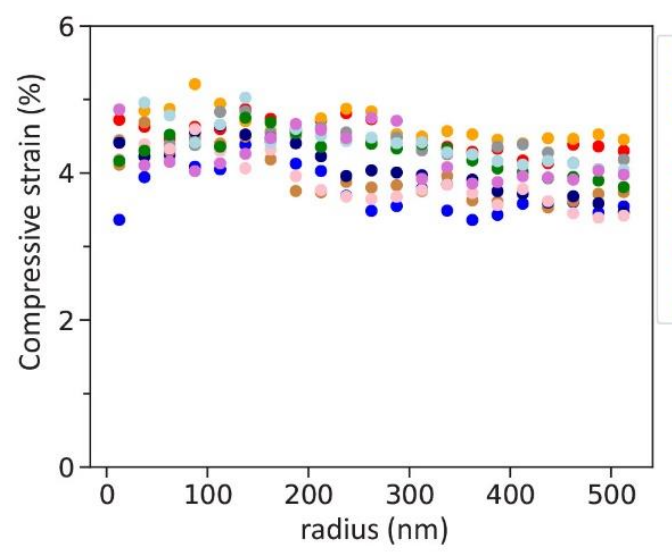

C

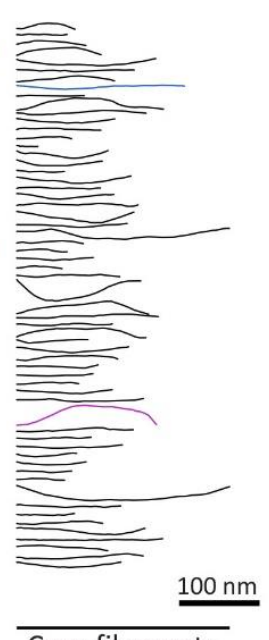

E

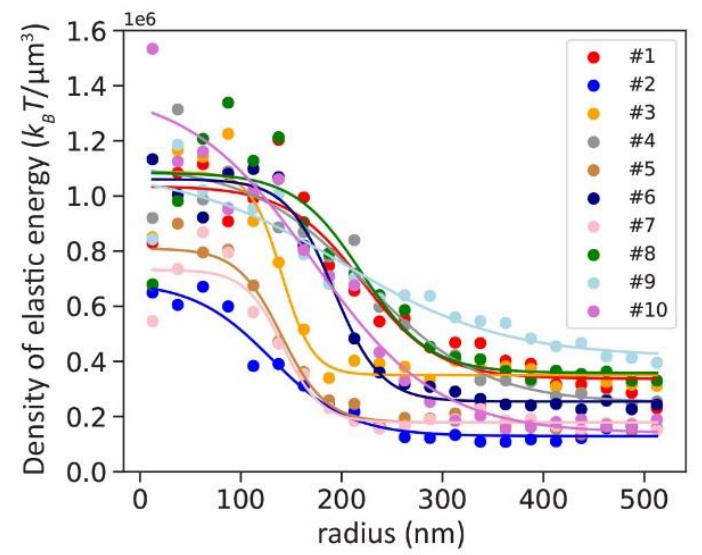

Fig. 3. Podosome filaments are compressed and store high elastic energy inside the core.

A: Perspective view of a cross-section through podosome \#1 displaying the mean curvature of the filaments. See also Movie S4.

B: Gallery of a random selection of actin filaments from podosome \#1. Filaments from the core (right) and outside the core (left) are shown.

C: Square of the local curvature along the filament length for the blue and purple filaments from the galleries of filaments outside (top) and inside (bottom) the core shown in (B).

D: Average compressive strain as a function of the radial distance from the core center for ten tomograms.

E: Density of elastic energy as a function of the radial distance from the core center for ten tomograms. 
A

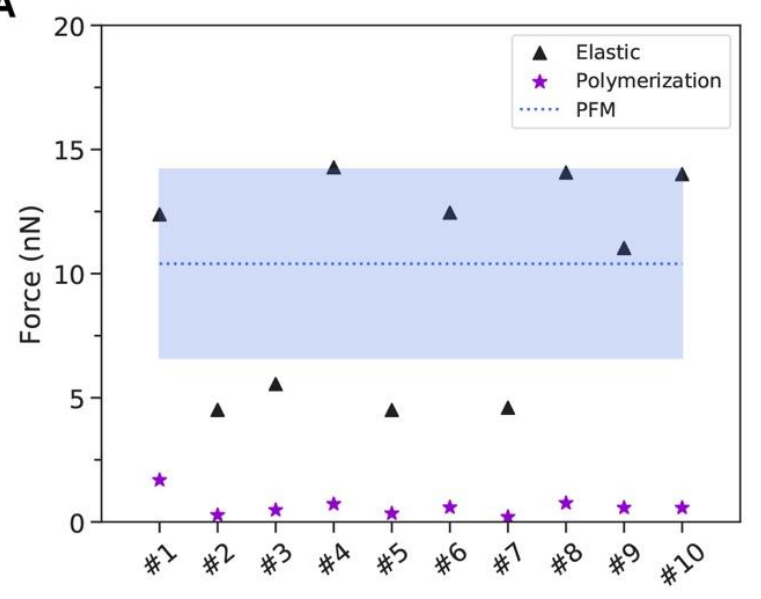

B

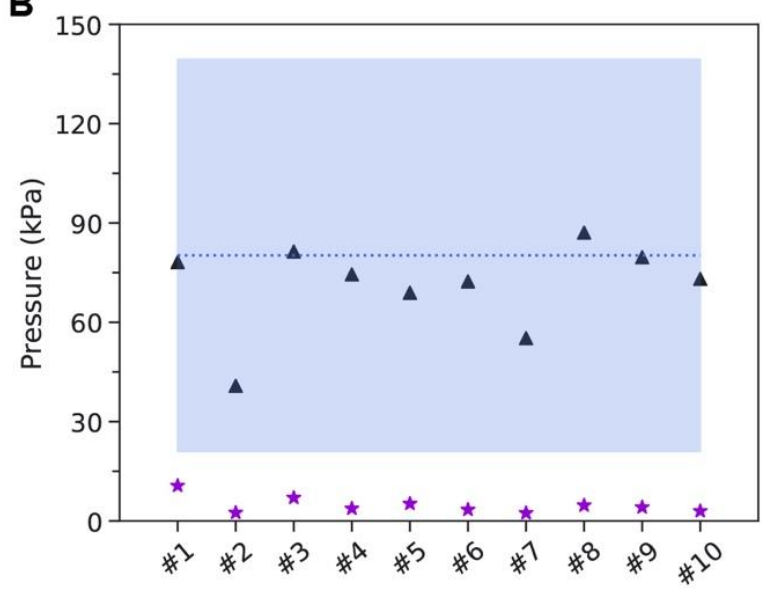

C
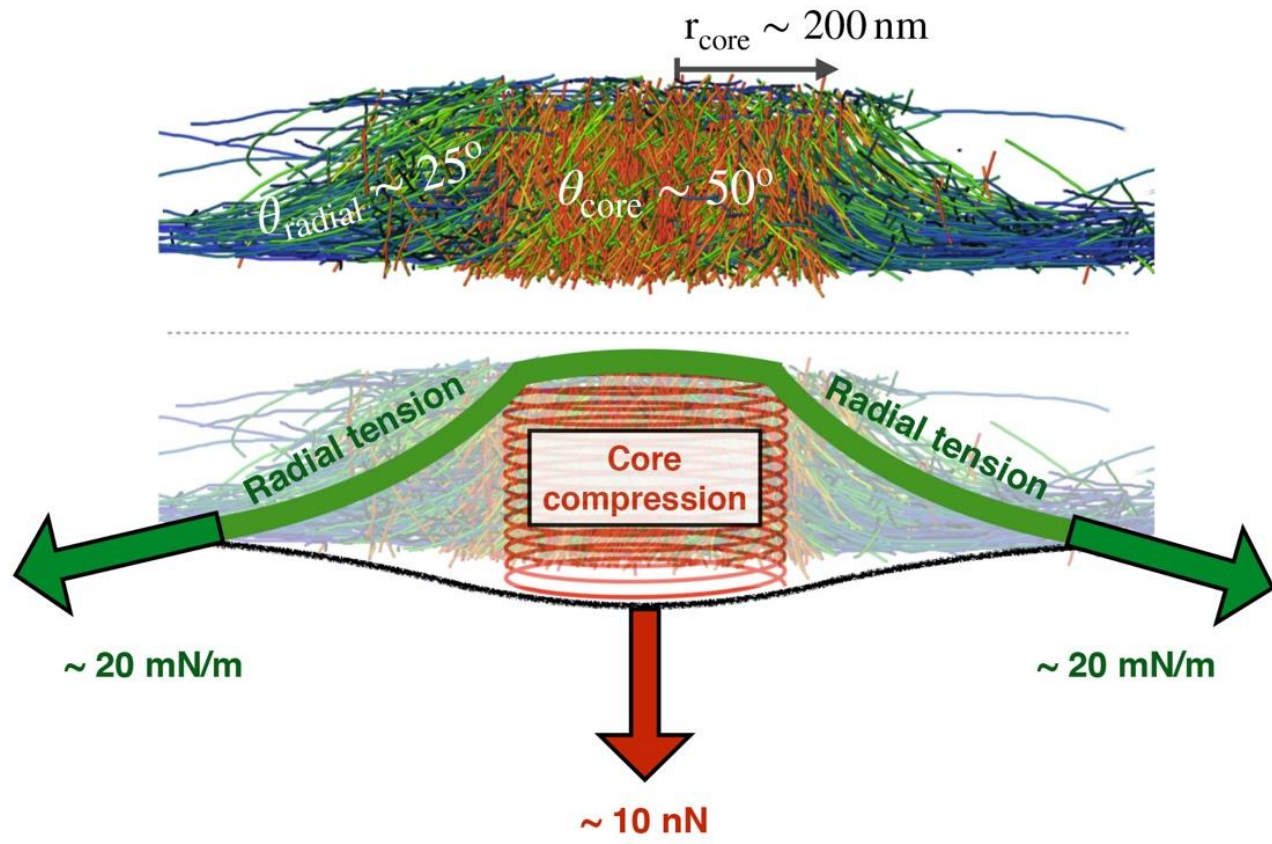

Fig. 4. The actin core generates elastic forces in the nanonewton range.

560 A: Comparison of the elastic force ("Elastic") generated by the core through the compression of the actin network (black triangle) with the estimated polymerization force ("Polymerization") generated at the core membrane (purple stars). The blue dashed line corresponds to the mean force derived from PFM measurements ("PFM") ${ }^{16}$ and the rectangular area filled in blue represents its standard deviation.

B: Same comparison plot as in (A) for the estimated pressure assuming a perfect circular shape for the podosome core (Methods).

C: Summary scheme showing the podosome organization revealed by cryo-ET, the elastic force resulting from core compression and the radial surface tension counterbalancing it. 


\section{Supplemental information}

\section{Movie S1. Native podosome architecture revealed by in situ cryo-ET.}

This movie shows the tomogram of the podosome presented in Figure $1 \mathrm{~A}-\mathrm{C}$ followed by its segmentation. Actin filaments are colored as a function of their orientation with respect to the basal membrane. Intermediate filaments are in brown and membranes in grey. Successive rotation, sectioning and zoom in the segmented volume allow the visualization of the dense organization of the actin filaments of the podosome core.

\section{Movie S2. Architecture of unroofed podosomes by cryo-ET.}

This movie shows the tomogram of unroofed podosomes presented in Figure S6 followed by its segmentation. Actin filaments are colored as a function of their orientation with respect to the basal membrane. Successive rotations, sectioning and zoom in the segmented volume reveal the nanoscale actin organization of neighboring podosomes.

\section{Movie S3. Visualization of the actin filaments in the vicinity of the plasma membrane beneath a podosome core.}

This movie shows the actin segmentation of the podosome presented in Figure 2A. Core filament segments in the vicinity of the basal membrane are colored as a function of their orientation. A series of rotations, zoomed and unzoomed sections through the segmented volume allow to visualize the number and orientation of the protrusive filaments at the core.

\section{Movie S4. Podosome actin filaments are bent.}

This movie shows the actin segmentation of the podosome presented in Figure 3A. Actin filaments are colored as a function of their mean curvature. Successive zoomed sections through the segmented volume allow to visualize the curvature of the filaments in a native podosome. 


\section{Average filament length: $<L>_{[r, r+\delta]}$}

\section{A}

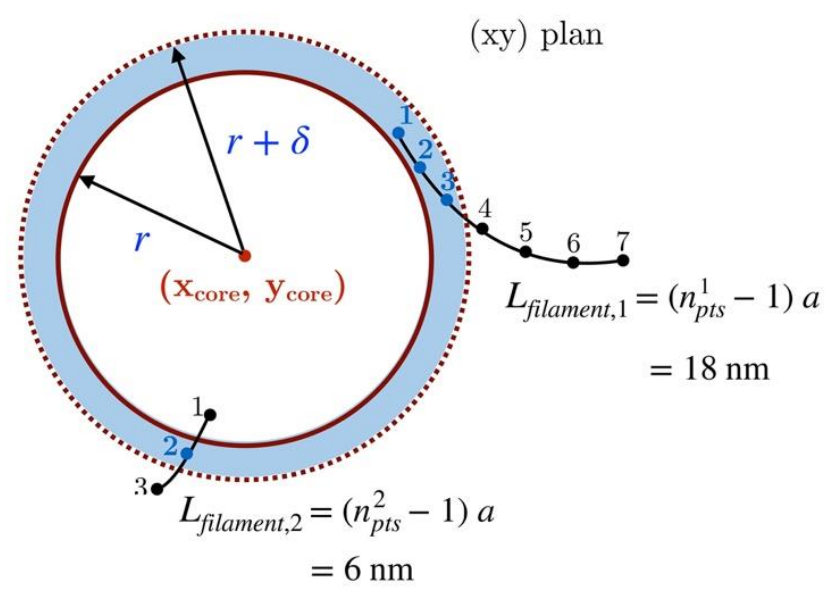

filament 1:

B

$$
\begin{aligned}
<L>_{[r, r+\delta]} & =\frac{1}{n_{p t s, i n}^{\text {tot }}} \sum_{i=1, \in[r, r+\delta]}^{n_{\text {fil }}} n_{p t s, \text { in }}^{i} L_{\text {filament }, i} \\
& =\frac{1}{4}\left[\frac{L_{\text {filament }, 1}+L_{\text {filament }, 1}+L_{\text {filament }, 1}}{\left.L_{\text {filament }, 2}\right]}=15 \mathrm{~nm}\right.
\end{aligned}
$$

598 Figure S1. Illustration of the computation of the average filament length as a function of 599 the radial distance.

600 A: For each distance bin $[r, r+\delta]$, the points inside the bins (shown in blue) are identified for each 601 actin filament (left). Each point is represented both in cartesian and radial coordinates (right).

602 B: The average length in the bin is the mean of the filament lengths weighted by their number of 603 points in the bin.

604 


\section{A}

\section{Average filament density: $d_{[r, r+\delta]}$}
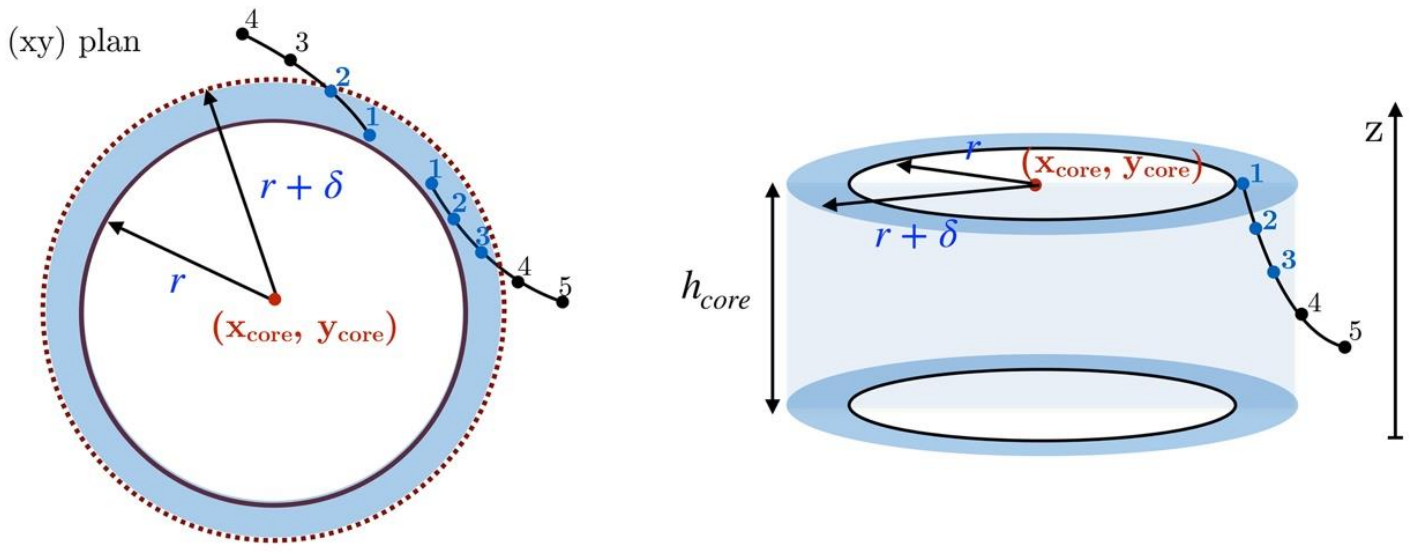

B

$$
\begin{aligned}
L_{t o t}[r, r+\delta] & =\sum_{\substack{i=1, \in[r, r+\delta] \\
n_{p t s, i n}^{i}>1}}^{n_{f i l}}\left(n_{p t s, i n}^{i}-1\right) \times a \\
& =3 \mathrm{~nm}+6 \mathrm{~nm}=9 \mathrm{~nm}
\end{aligned}
$$
608 the radial distance.

609 A: For each bin $[r, r+\delta]$, the points inside the bins (shown in blue) are identified for each actin 610 filament.

611 B: The total actin length is calculated in each bin and divided by the bin volume to compute the 612 density.

613

614 
A

Local filament orientation:
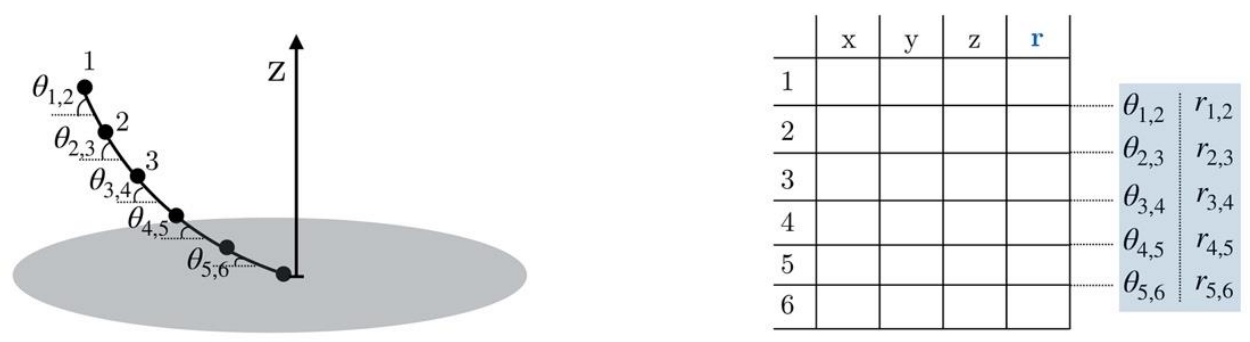

$\theta_{i, i+1}=\frac{180}{\pi}\left|\arcsin \left\{\frac{z_{i+1}-z_{i}}{\sqrt{\left(\left(x_{i+1}-x_{i}\right)^{2}+\left(y_{i+1}-y_{i}\right)^{2}+\left(z_{i+1}-z_{i}\right)^{2}\right)}}\right\}\right|$

B

$$
\begin{aligned}
& \text { Average filament orientation: }\langle\theta\rangle_{[r, r+\delta]} \\
& <\theta>_{[r, r+\delta]}=\frac{1}{n_{p t s, \text { ton }}^{\text {tot }}} \sum_{i \in[r, r+\delta]} \theta_{i}
\end{aligned}
$$

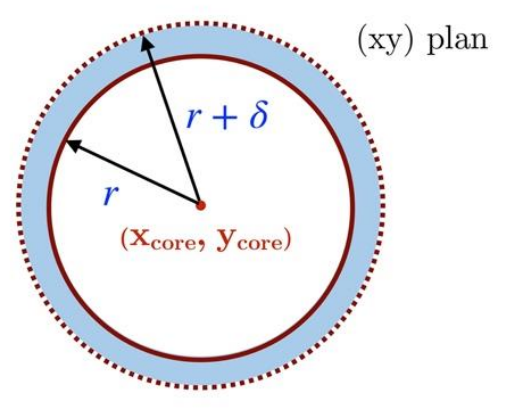

616 Figure S3. Illustration of the computation of the average filament orientation as a function 617 of the radial distance.

618 A: To each segment between two filament points is associated an angle with respect to the membrane plane.

620 B: The average filament orientation in a bin is the mean of the orientations of all segments inside the bin. 
bioRxiv preprint doi: https://doi.org/10.1101/2021.04.13.439622; this version posted April 14, 2021. The copyright holder for this preprint (which was not certified by peer review) is the author/funder, who has granted bioRxiv a license to display the preprint in perpetuity. It is made available under aCC-BY-NC-ND 4.0 International license.

A
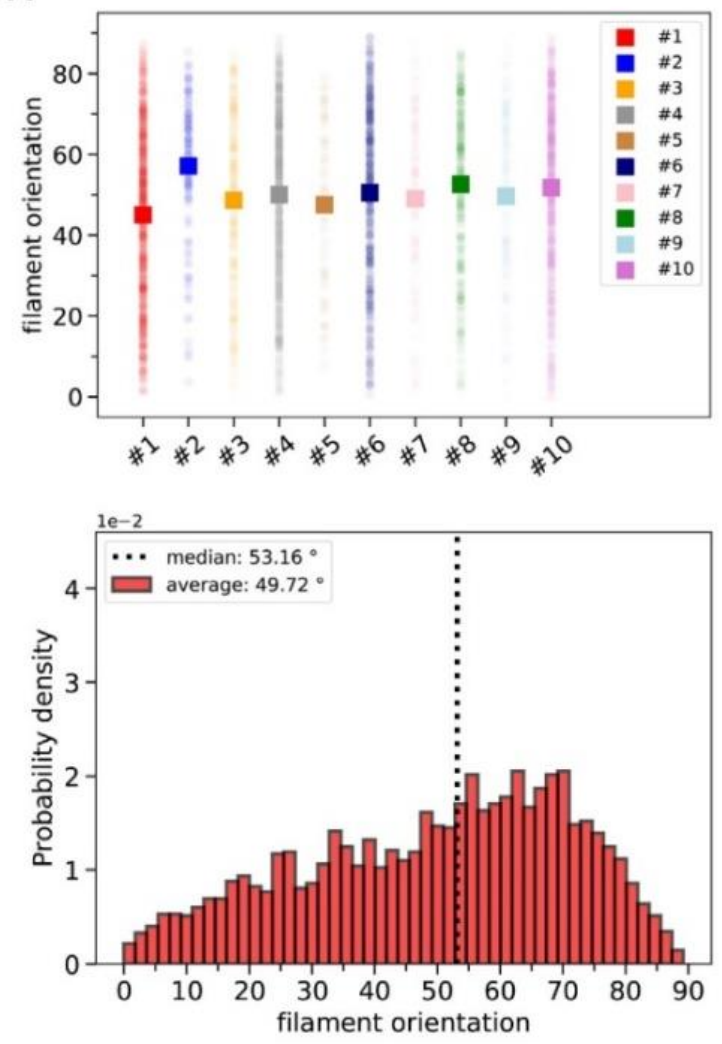

C
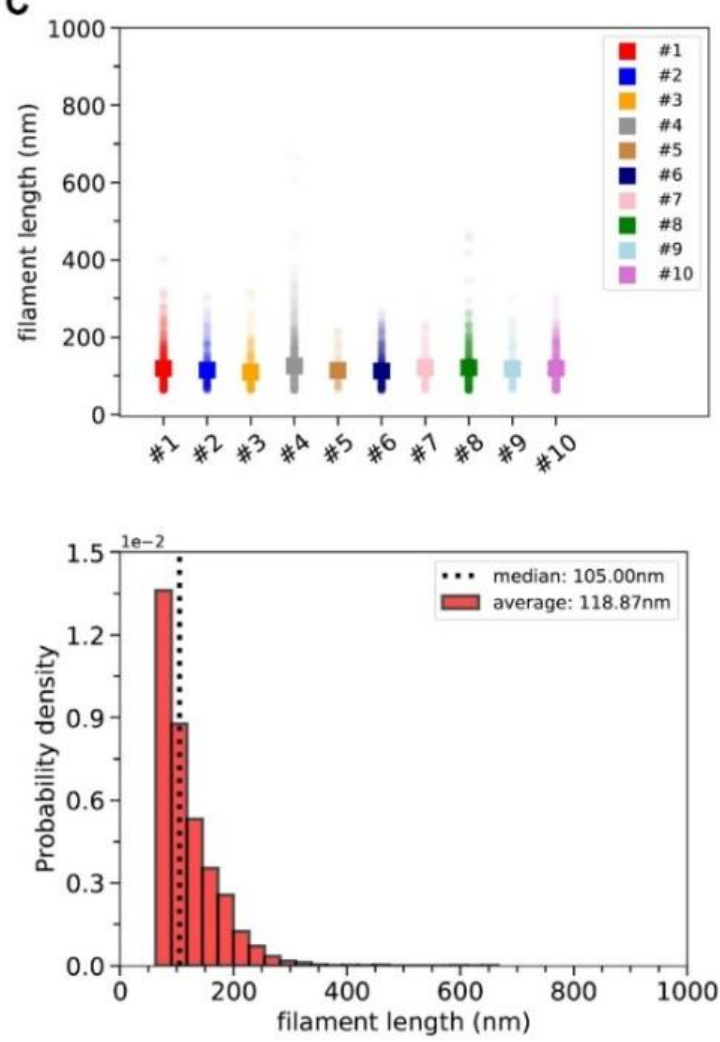

B
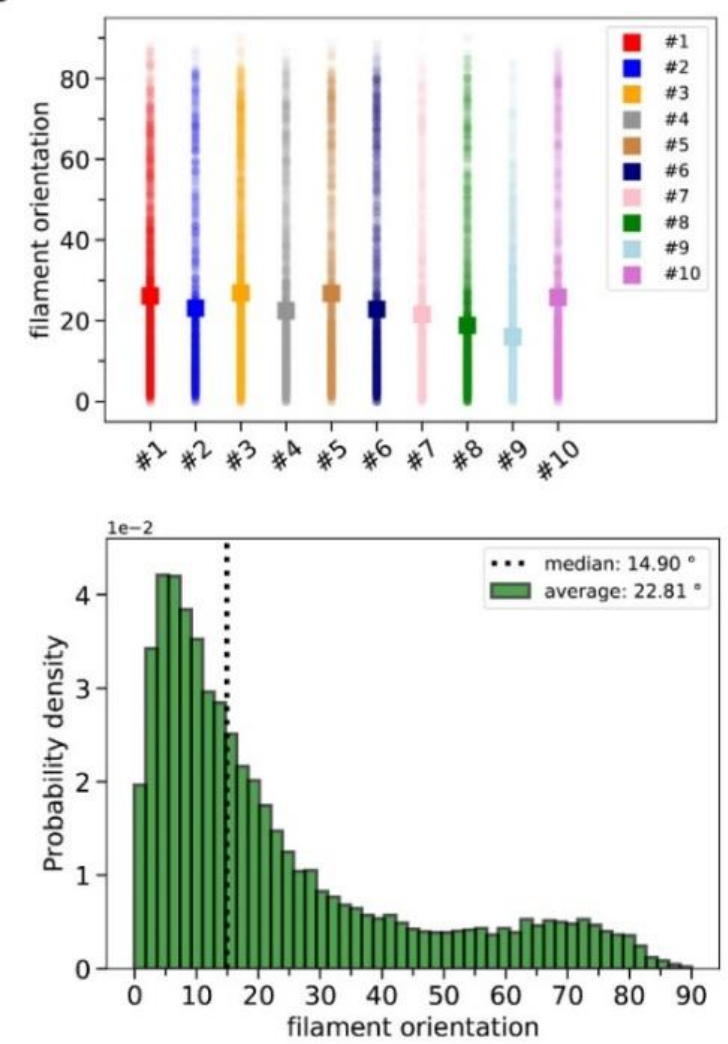

D
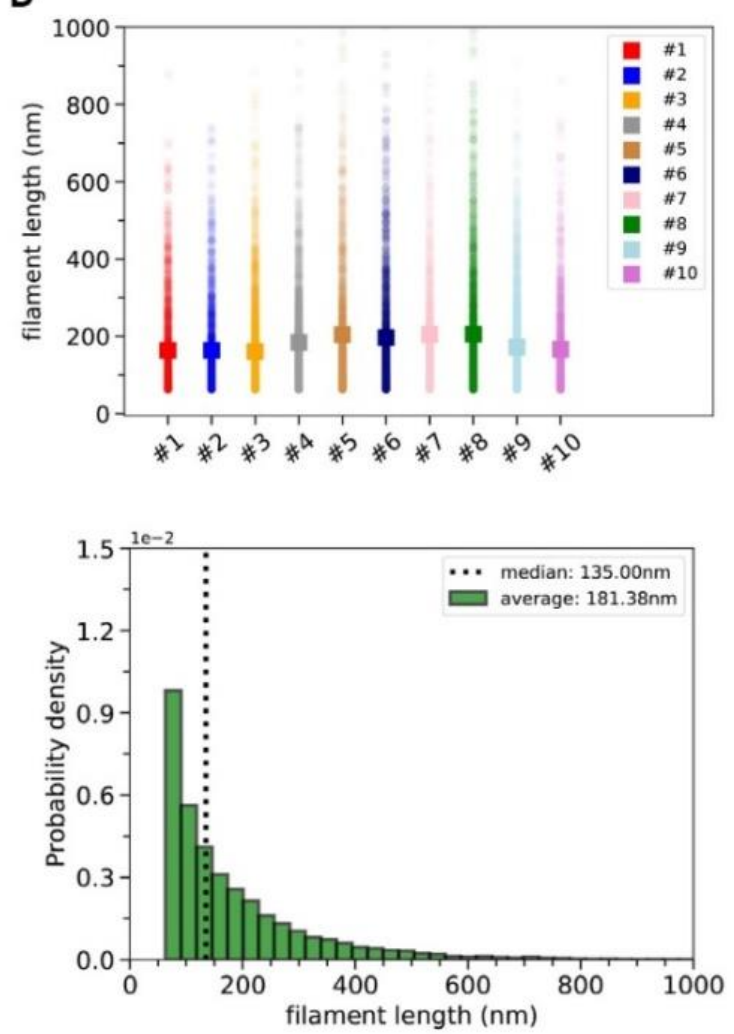
629 Figure S4. Orientation and length distributions of the innermost and outermost filaments, 630 respectively, in ten podosomes.

631 A: (Top) Mean orientation of the innermost filaments within the radial distance range $r_{\epsilon}\left[r_{0}-r_{s}, r_{0}\right]$. $632 r_{0}$ and $r_{s}$ are the inflection point and transition range parameters, respectively, and were obtained 633 from the fit of the orientation as a function of $r$ for each tomogram. (Bottom) Corresponding 634 distribution obtained by merging all the data from the top panel into a single dataset. The vertical 635 dashed line indicates the median value of the dataset.

636 B: Same distributions for the outermost filaments, i.e. within the radial distance range $r \epsilon$ $637 \quad\left[r_{0}+r_{s}, r_{\max }\right]$ where $r_{\max }$ is the highest radial distance between a point filament and the core center. 638 C-D: Same plots as in A-B for the filament length. 
A
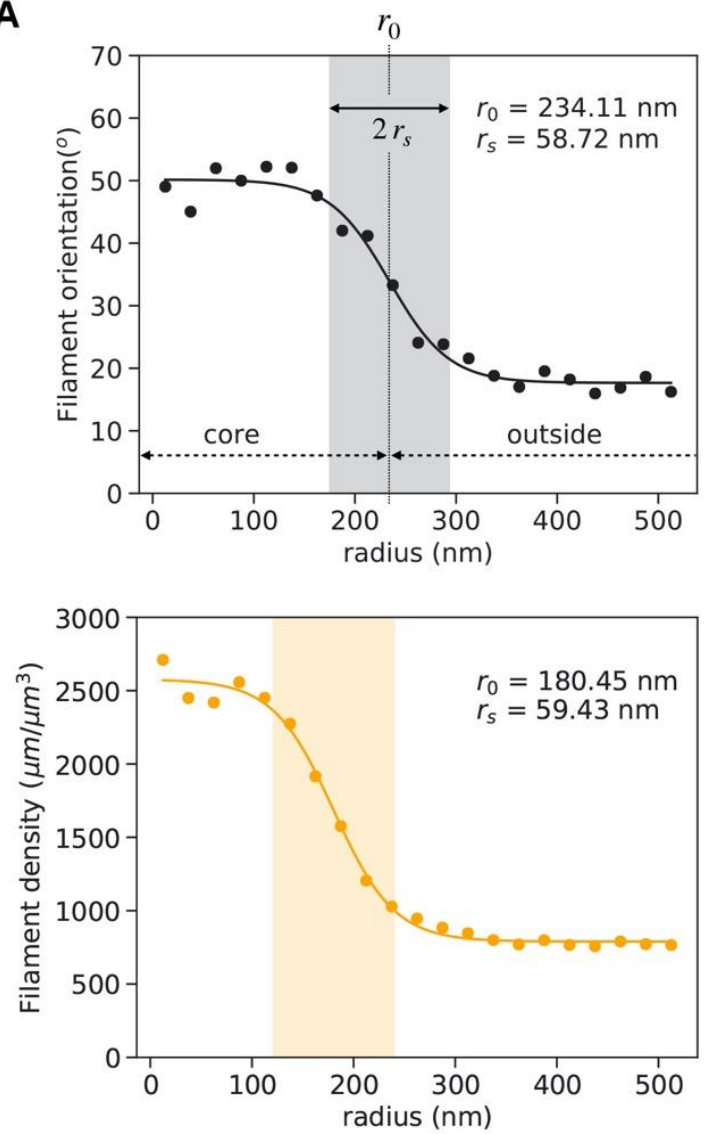

B

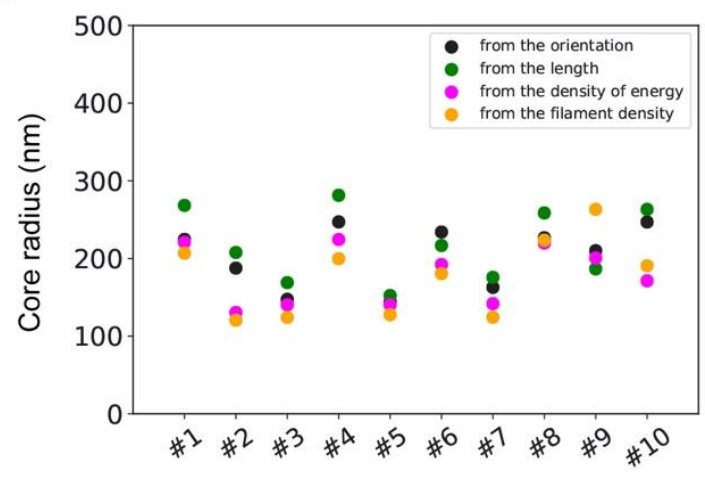

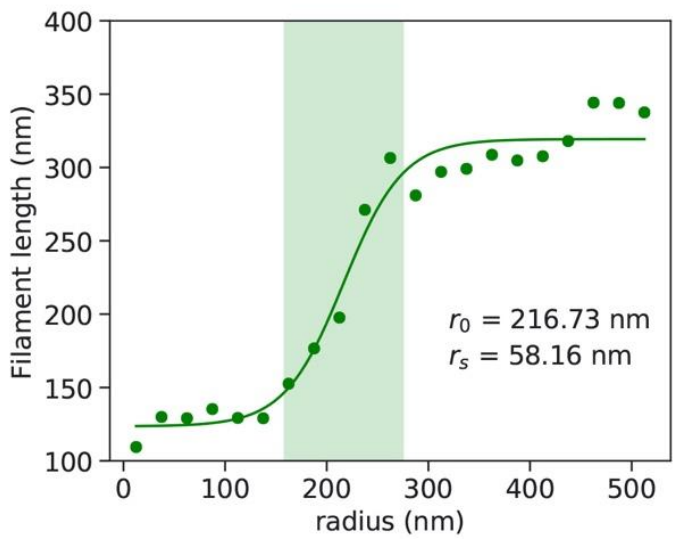
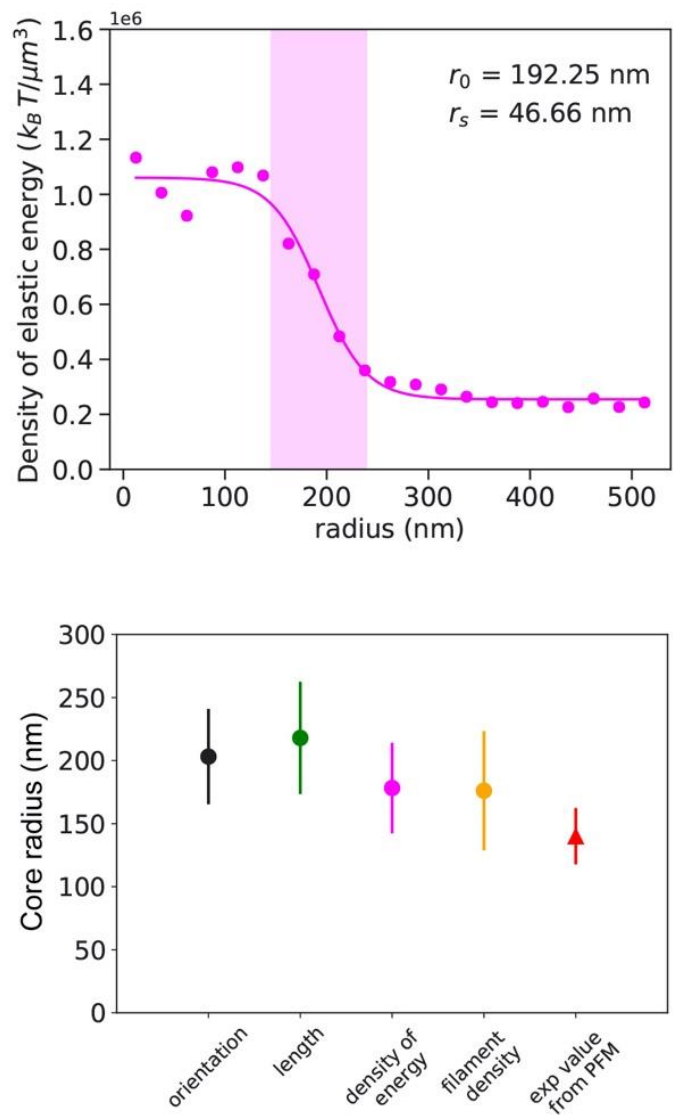

639

640

641

642

643

644

645

646

647

648

Figure S5. Estimation of the core radius from the distribution of each parameter as a function of the distance to the core center.

A: Filament orientation, length, actin density and density of energy for podosome \#6 with their respective fits are shown. The parameters $r_{0}$ and $r_{s}$ and the transition (colored) zone are indicated.

B: (Left) Comparison of the core radius values obtained from the fits of the different parameters as a function of the radial distance for the ten tomograms. (Right) Average radius of the podosome core estimated from the quantitative analysis of the cryo-ET data and compared to the reported value from PFM (mean \pm s.d.). 

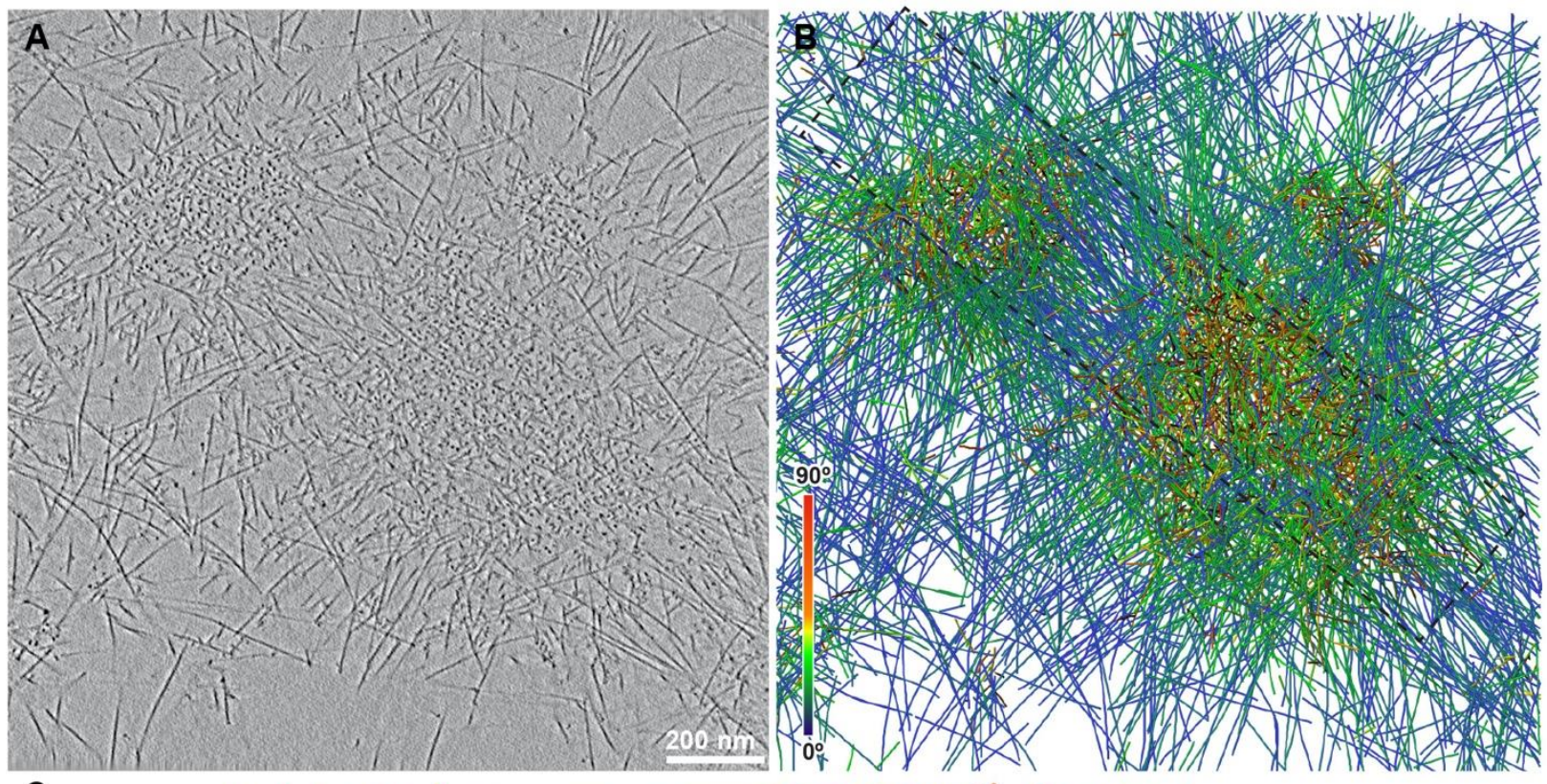

C

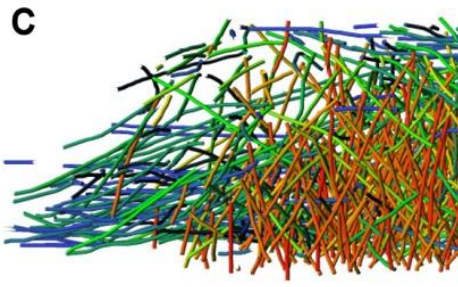


Compressive strain: $\epsilon$

$$
\epsilon=1-\frac{c}{L}
$$
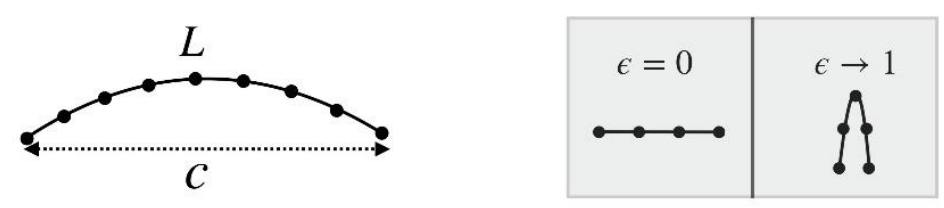

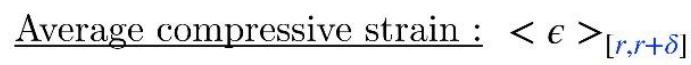

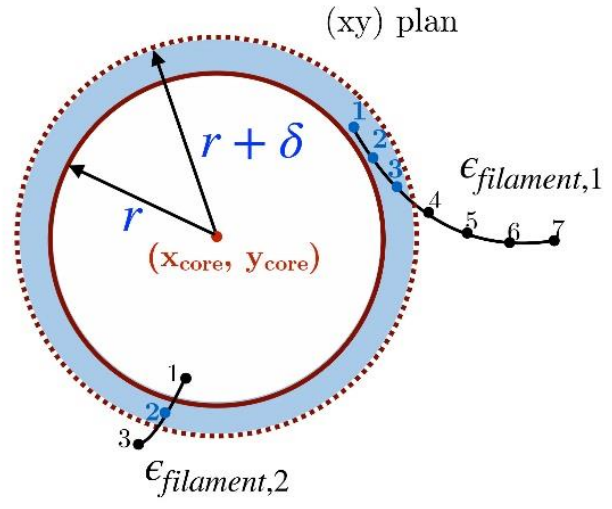

$$
\begin{aligned}
<\epsilon>_{[r, r+\delta]}= & \frac{1}{n_{p t s, i n}^{\text {tot }}} \sum_{i=1, \in[r, r+\delta]}^{n_{f i t}} n_{p t s, \text { in }}^{i} \epsilon_{f i l a m e n t, i} \\
& =\frac{1}{4}\left[3 \times \epsilon_{\text {filament }, 1}+1 \times \epsilon_{\text {filament }, 2}\right]
\end{aligned}
$$

661 Figure S7. Illustration of the computation of the average compressive strain as a function 662 of the radial distance.

663 A: The compressive strain is computed as 1 minus the ratio between the end-to-end distance, $c$, 664 and the filament length, $L$. Thus, a straight filament has a zero strain.

665 B: The average compressive strain in a bin is computed as the mean of the compressive strains 666 weighted by the number of filament points in the bin.

667 
A

Elastic energy per filament: $u_{\text {elastic }}$

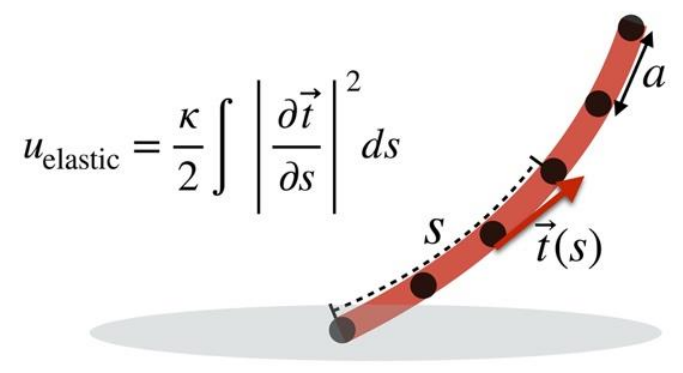

B

Elastic energy per volume: $\quad u_{[r, r+\delta]}$

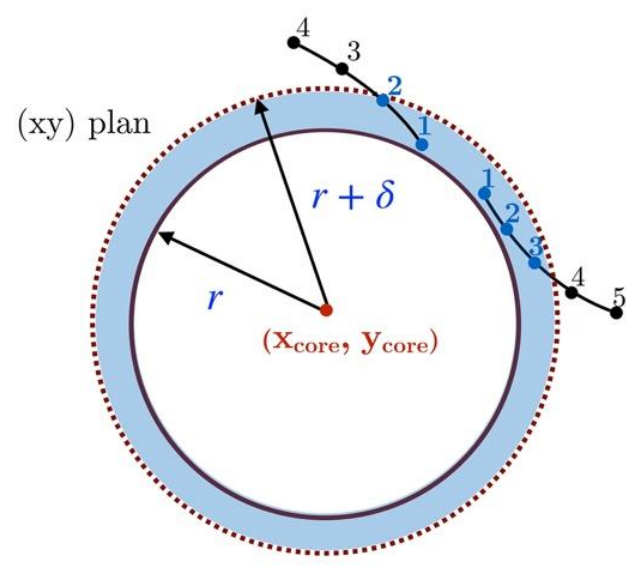

$E_{t o t}[r, r+\delta]:$ sum of local energy on all points in the interval $[r, r+\delta]$

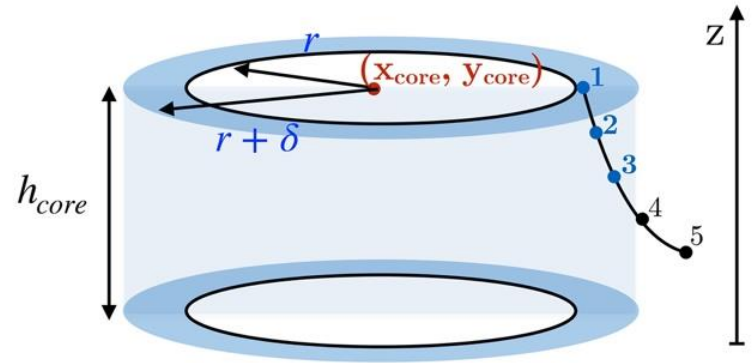

$$
u_{[r, r+\delta]}=\frac{E_{t o t}[r, r+\delta]}{\pi h_{\text {core }}\left((r+\delta)^{2}-r^{2}\right)}
$$

Figure S8. Illustration of the computation of the elastic energy per volume as a function of the radial distance.

672 A: Definition of the elastic energy of a filament as the integral of the squared curvature over the 673 filament length. the elastic energy of all the filament points in the bin divided by the volume. 